\title{
A Human-in-the-loop Evaluation of a Coordinated Arrival/Departure Scheduling Operations for Managing Departure Delays at LaGuardia Airport
}

\author{
Paul U. Lee ${ }^{1}$, Nancy M. Smith ${ }^{2}$, Nancy Bienert ${ }^{3}$, Connie Brasil ${ }^{1}$, Nathan Buckley ${ }^{3}$, Eric Chevalley ${ }^{4}$, \\ Jeffrey Homola ${ }^{5}$, Faisal Omar ${ }^{5}$, Bonny Parke ${ }^{4}$, and Hyo-Sang Yoo ${ }^{5}$ \\ NASA Ames Research Center, Moffett Field, CA, 94035
}

\begin{abstract}
LaGuardia (LGA) departure delay was identified by the stakeholders and subject matter experts as a significant bottleneck in the New York metropolitan area. Departure delay at LGA is primarily due to dependency between LGA's arrival and departure runways: LGA departures cannot begin takeoff until arrivals have cleared the runway intersection. If one-in one-out operations are not maintained and a significant arrival-to-departure imbalance occurs, the departure backup can persist through the rest of the day. At NASA Ames Research Center, a solution called "Departure-sensitive Arrival Spacing" (DSAS) was developed to maximize the departure throughput without creating significant delays in the arrival traffic. The concept leverages a Terminal Sequencing and Spacing (TSS) operations that create and manage the arrival schedule to the runway threshold and added an interface enhancement to the traffic manager's timeline to provide the ability to manually adjust inter-arrival spacing to build precise gaps for multiple departures between arrivals. A more complete solution would include a TSS algorithm enhancement that could automatically build these multi-departure gaps. With this set of capabilities, inter-arrival spacing could be controlled for optimal departure throughput. The concept was prototyped in a human-inthe-loop (HITL) simulation environment so that operational requirements such as coordination procedures, timing and magnitude of TSS schedule adjustments, and display features for Tower, TRACON and Traffic Management Unit could be determined. A HITL simulation was conducted in August 2014 to evaluate the concept in terms of feasibility, controller workload impact, and potential benefits. Three conditions were tested, namely a Baseline condition without scheduling, TSS condition that schedules the arrivals to the runway threshold, and TSS+DSAS condition that adjusts the arrival schedule to maximize the departure throughput. The results showed that during high arrival demand period, departure throughput could be incrementally increased under TSS and TSS+DSAS conditions without compromising the arrival throughput. The concept, operational procedures, and summary results were originally published in $\mathrm{ATM2015}^{1}$ but detailed results were omitted. This paper expands on the earlier paper to provide the detailed results on throughput, conformance, safety, flight time/distance, etc. that provide extra insights into the feasibility and the potential benefits on the concept.
\end{abstract}

\section{Introduction}

LaGuardia airport (LGA) has one of the largest taxi-out delays in the National Airspace System (NAS). Departure delay at LGA is primarily due to the dependency between LGA's arrival and departure runways: LGA departures cannot begin take-off until arrivals have cleared the runway intersection. LGA experiences high arrival

\footnotetext{
${ }^{1}$ Senior Research Associate, Human Systems Integration Division, NASA ARC; paul.u.lee@nasa.gov

${ }^{2}$ Research Psychologist, Human Systems Integration Division, NASA ARC; nancy.m.smith-1@nasa.gov

${ }^{3}$ Research Associate, Human Systems Integration Division, SJSU/NASA ARC, MS 262-4

${ }^{4}$ Senior Research Psychologist, Human Systems Integration Division, SJSU/NASA ARC, MS 262-4

${ }^{5}$ Research Engineer, Human Systems Integration Division, SJSU/NASA ARC, MS 262-4

1
}

American Institute of Aeronautics and Astronautics 
and departure demand throughout the day, high gate utilization, limited airport surface area, and a rapid turnaround time per flight. If one-in one-out operations (one arrival followed by a departure) are not maintained, a significant arrival-to-departure imbalance occurs and the departure backup can persist throughout the rest of the day. The impact can include surface congestion that delays gate access for arrivals and turnaround time, causing ripple effects throughout the NAS. The data from the FAA's Aviation System Performance Metrics (ASPM) website confirmed that the LGA's taxi-out delay (average taxi-out time - average unimpeded taxi-out time) was consistently the highest in the country, averaging 13.14 minutes per flight between 2010 and 2012. This translates to increased (taxi) fuel costs and transit times per flight. A solution to this problem could better accommodate fluctuations in departure demand, improve aircraft turnaround time and reduce the risk of surface gridlock.

Although departure delay problems due to arrival-departure dependencies are most acute for LGA, similar problems exist at other high demand airports that have either 1) intersecting arrival-departure runways or 2) mixed arrival-departure mode operations on a single runway. Past research efforts, both in the U.S. and in Europe, have developed operational tools and concepts that propose to improve the departure throughput and/or reduce delays by adjusting the arrival spacing. ${ }^{2,3,4}$ Leveraging the insights from the past research, a new Trajectory-Based Operation (TBO) solution called "Departure-sensitive Arrival Spacing" (DSAS) was developed at NASA Ames Research Center to address the LGA departure issues. The concept uses Terminal Sequencing and Spacing (TSS) operations ${ }^{5,6}$ to manage the actual runway threshold times for arrivals. An interface enhancement to the TSS scheduler provides the ability to manually adjust inter-arrival spacing to build precise gaps for multiple departures between arrivals. The concept was prototyped in a human-in-the-loop (HITL) simulation environment in August 2014 to evaluate the concept in terms of feasibility, controller workload impact, and potential benefits. The results of the study was published in ATM2015 ${ }^{1}$ but due to the page limits, the detailed results on throughput, conformance, safety, flight time/distance, etc. were omitted in the earlier paper. Therefore, the purpose of this paper is to provide complementary analyses and more detailed results from the HITL simulation that provide greater insight into the overall concept benefits and feasibility.

\section{Operational Concept for LaGuardia Arrival-Departure Operations}

In current operations, LGA regularly has high arrival and departure rates that begin early morning and persist consistently throughout the day, frequently reaching 38 or 39 arrivals per hour during afternoon and evening. In general, under Visual Flight Rules (VFR), a $2.5 \mathrm{~nm}$ inter-arrival spacing at the runway threshold is observed, allowing for a departure between each arrival pair while maintaining a high arrival rate. However, VFR operations do not guarantee this minimum inter-arrival spacing, thus a loss of departure slots and buildup of departure queues may occur. Paradoxically, the feedback from the New York subject matter experts (SMEs) was that sometimes the TRACON controllers reduce the arrival rates when they perceive excessive departure delays, thinking that the lower arrival rates will allow a greater number of departures to take-off. However, the reduced arrival rates could increase the inter-arrival spacing but not enough to allow two-for-one departure-arrival operations, which can further increase both arrival and departure delays.

DSAS solution proposes to relieve the departure delays without significantly impacting the arrival capacity. This can be accomplished by 1) creating a consistent inter-arrival spacing that ensures one-for-one departure/arrival operations and 2) slightly modifying the arrival schedule to create inter-arrival spacing that is optimal for one, two or more departures.

\section{A. TSS Operations to Ensure One-for-one Operations}

In order to ensure one-for-one operations (i.e. one departure for one inter-arrival spacing), TSS is used to create and deliver an arrival schedule that would minimize the loss of departure slots due to inconsistent inter-arrival spacing. TSS enhances the existing meter fix scheduler to create a Terminal Metering schedule. It utilizes new area navigation (RNAV) enabled descent procedures that extend from En Route airspace to the runway threshold. Figure 1 shows an example of a TSS schedule. The right side of the graph shows the Scheduled Times of Arrival (STAs) to the runway threshold using wake vortex spacing. The left side of the graph shows the Estimated Times of Arrival (ETAs). The numbers next to an aircraft's STA indicate the delay that the aircraft needs to absorb (in minutes) in order to conform to their STA. 


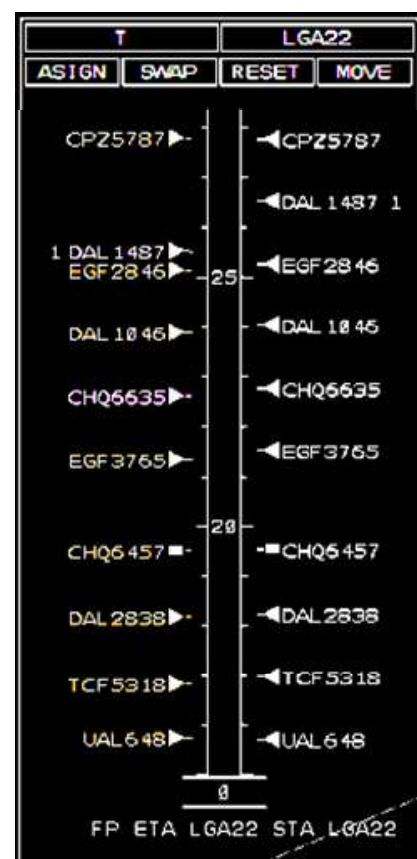

Figure 1. An example of TSS schedule

A TSS schedule to the runway threshold provides spacing that is enough to fit at least one departure between an arrival pair, provided that the arrivals can conform to the schedule. The TRACON controllers achieve the schedule conformance using TSS slot markers that indicated where an aircraft was scheduled to be along its RNAV route. These slot markers represented the current "ideal" position and the indicated air speed of the aircraft as it descended from the meter fix to the runway along the 4-D path defined by its RNAV descent procedure and assigned STA. Figure 2 shows a TRACON controller display with slot marker circles, as well as the charted air speed for the route. Controllers issued speed or route amendments to put the aircraft in their slot markers to conform to their STAs.

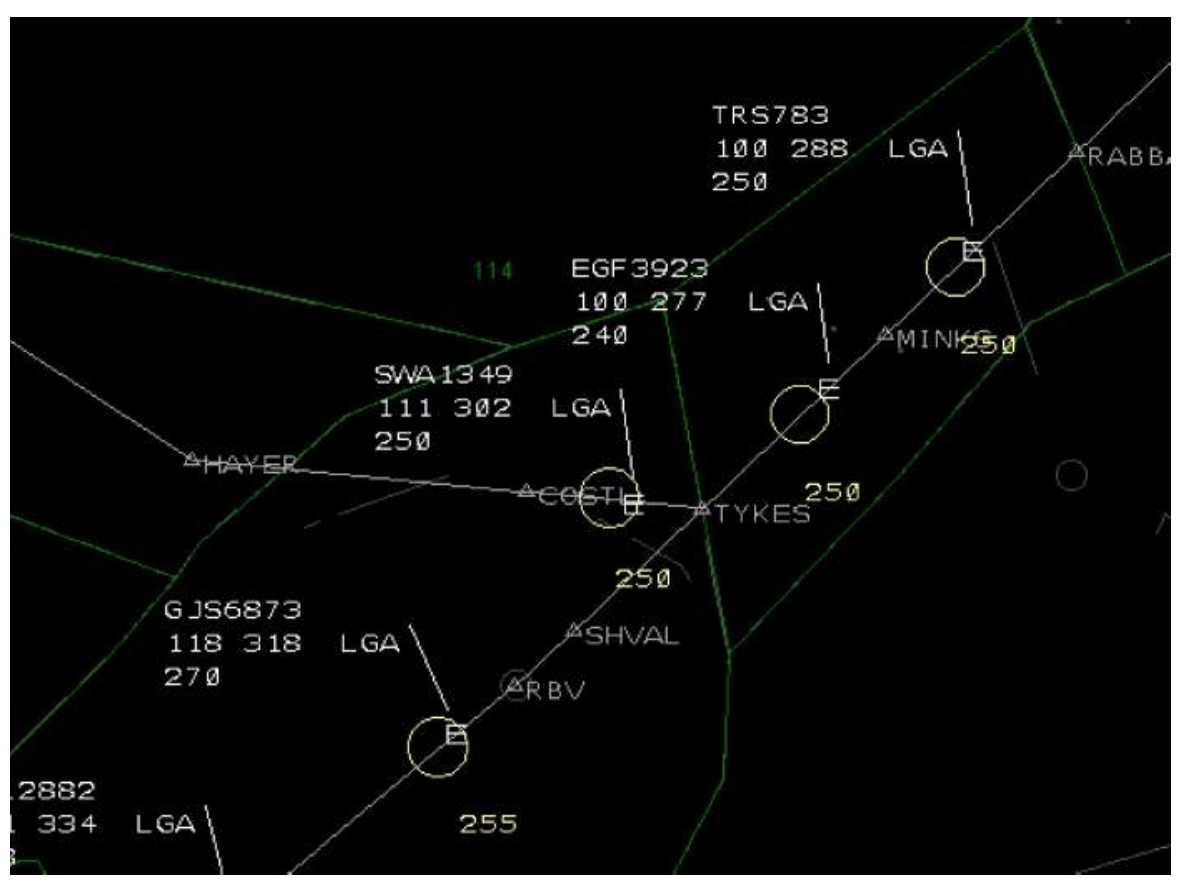

Figure 2. An example of TSS slot markers 


\section{B. DSAS Operations for Increasing Departure Throughput}

Even with the TSS scheduler, the inter-arrival spacing may not be optimal for departures, as it can often be greater than the spacing needed to allow one departure between an arrival pair but not enough to allow multiple departures. If the arrival spacing can be adjusted to match the exact spacing needed for one or more departures per arrival pair, more departures could be cleared given the same arrival demand ${ }^{7}$.

DSAS proposes to adjust the TRACON schedule prior to TRACON entry to optimize the inter-arrival spacing for the departures. The DSAS concept assumes a new Traffic Management Unit (TMU) Planner position (called "Planner" in our simulation), who can modify the schedule after the schedule is frozen and before the controllers start moving the aircraft to meet their STAs. The Planner can adjust the schedule by assigning larger inter-arrival spacing to allow multiple departures. Figure 3 illustrates a schematic example of how the schedule could be adjusted. The left graph shows an example of an arrival schedule with only the TSS schedule. The inter-arrival spacing may be greater than the minimum wake vortex spacing (set to 75 seconds in this condition) but the spacing is not large enough for multiple departures per arrival pair. Using DSAS operations, as shown on the right, the arrival schedule is modified to create 75 seconds minimum spacing for the first four arrivals, which then creates a larger 120 seconds spacing between the arrival 4 and 5, which is enough spacing to allow two departures in that gap.
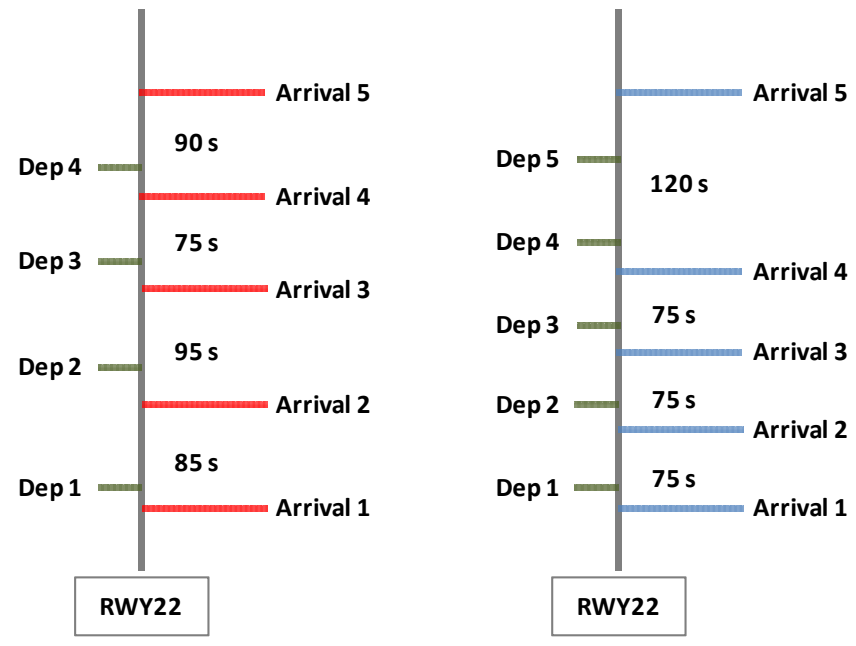

Figure 3. Original arrival schedule on the left graph (in red) and the arrival schedule modified with DSAS on the right graph (in blue)

\section{Human-in-the-loop Evaluation of the DSAS Operations}

A simulation study was conducted in August 2014 to evaluate the concept in terms of feasibility, controller workload impact, and potential benefits. New Optimized Profile Descent (OPD) routings, procedures, and prototype decision support tools were designed to support the TSS/DSAS operations.

\section{A. Experiment Design}

The experiment was a within-subjects $3 \times 2$ design. The two independent variables were operational environment: Baseline, TSS, and TSS+DSAS (or DSAS for short) and traffic levels: Moderate and High traffic. Each condition was run once, consisting of six total runs. The Baseline condition assumed current day tools but with newly designed routes and airspace. The TSS and DSAS conditions included what was described in the previous TSS and DSAS operations sections.

\section{B. Participants}

Eight retired FAA personnel served as controller participants. The four En Route controller participants were from Oakland Center (ZOA). The four TRACON and tower controller participants worked in different TRACON facilities (e.g. Northern California, Dayton, and Miami) as well as tower and center facilities. Their air traffic control

4

American Institute of Aeronautics and Astronautics 
experience ranged from 21 to 31 years and their retirement dates from 1 to 7 years ago. The other participants were retired Supervisory Traffic Management Coordinator from ZOA, Front Line Managers (FLMs) from Washington Center (ZDC) and New York TRACON (N90), who played the role of a TMU Planner, an En Route FLM and a TRACON Sequencer, respectively. One of the researchers also participated in the study as a tower cab coordinator. All of the simulated aircraft were flown by pseudo-pilots, who were active commercial pilots or students from the aviation department at San Jose State University.

\section{Airspace and Runway Configurations}

The simulated airspace consisted of a subset of the New York TRACON (N90) sectors that feed traffic into LGA Airport. Figure 4 shows the two feeder sectors (i.e., Empyr and Haarp) and one final sector (Final). These sectors have been modified from current sector configurations in order to accommodate OPD routes that have been newly designed for this study.

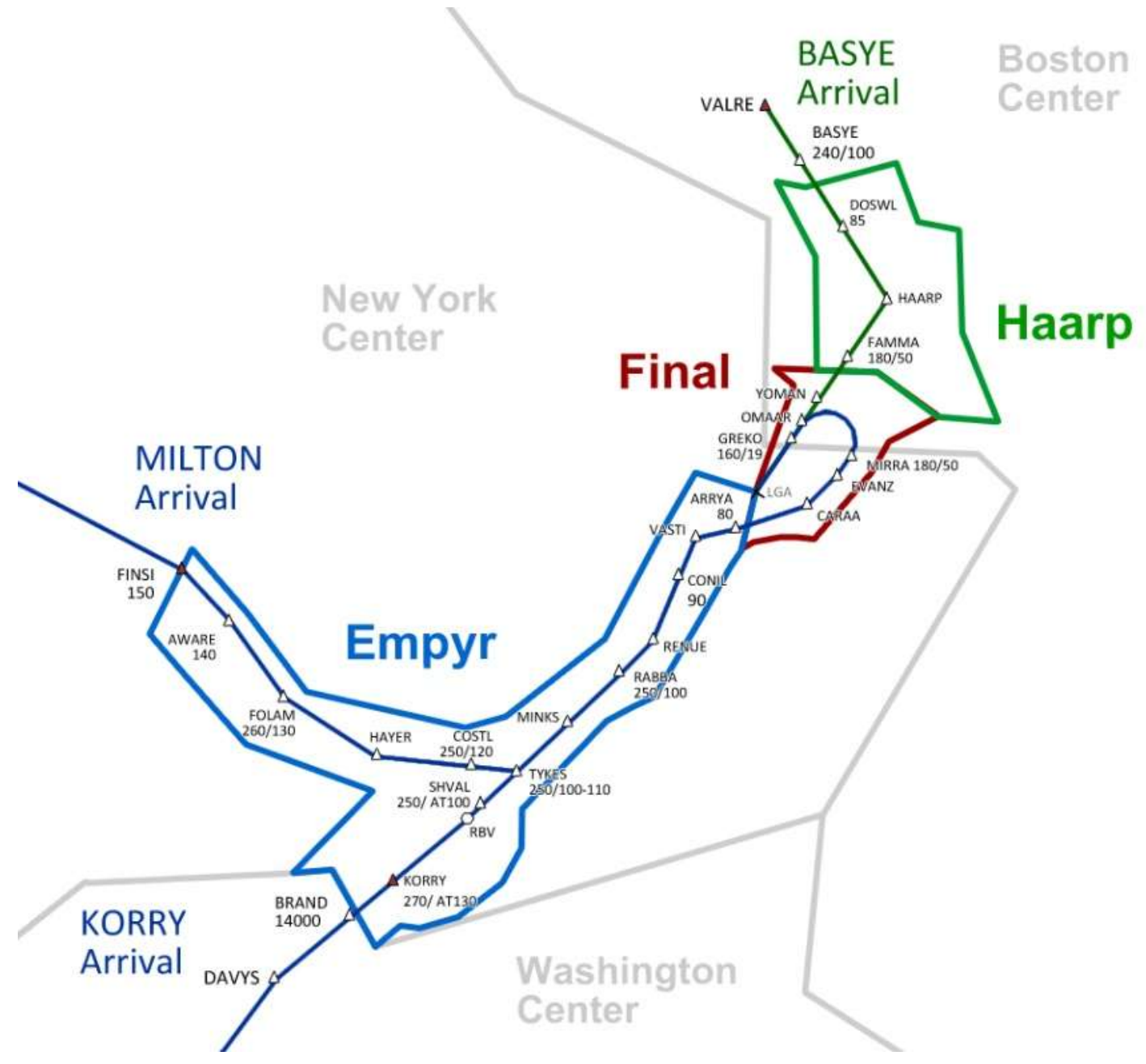

Figure 4. Simulated airspace: two feeder and one final sectors to LGA

The most common operations in LGA assign one of these runways as the departure runway and the other as the arrival runway. Based on ASPM data, the four most commonly used runway configurations (in order of frequency) are 22|13 (i.e., landing runway 22 and departing runway 13), 31|4, 22|31, and 4|13 (see Figure 5). 


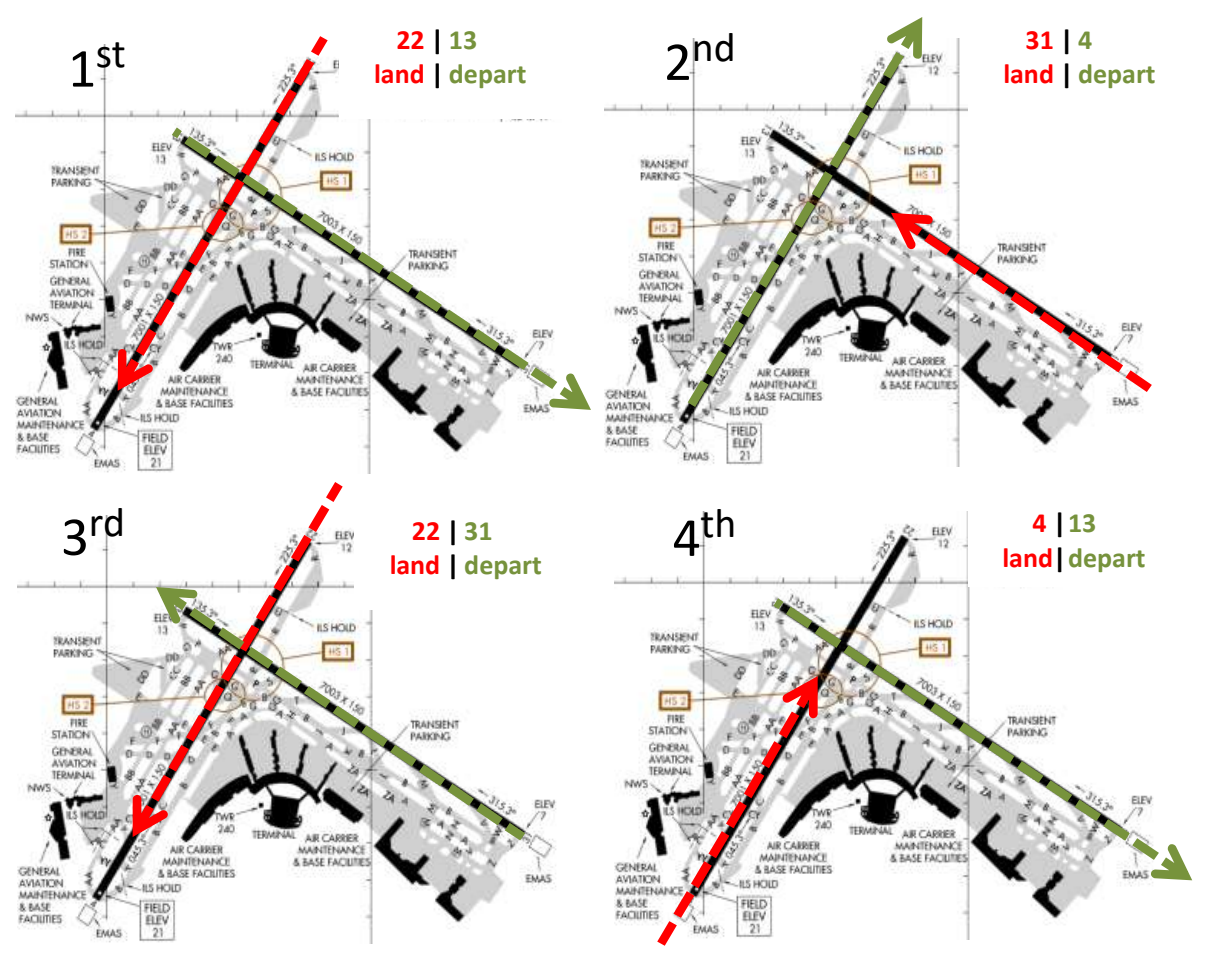

Figure 5. Four most commonly used runway configurations at LGA

For this study, the 22|31 configuration was chosen from the four most common configurations illustrated in Figure 5. This required a fairly precise delivery of the arrivals on schedule in order to not miss the departure slots, thereby creating a challenging problem for evaluation. The reason is that in a crossing runway configuration, the controller cannot clear the departure until the arrival passes the runway crossing point. However, the departure then also needs to pass the runway crossing point before the trailing arrival reaches the runway threshold. These interactions mean that whenever the runway crossing point is farther from the departure point, there is less margin of error for the inter-arrival spacing or the departure takeoff time accuracy before losing the departure slot. The 22|31 and $31 \mid 4$ configurations have these runway characteristics.

In addition, 22|31 required a last-minute tactical adjustment of the schedule to create the spacing for B757 departures, which provided an additional challenge for the DSAS concept. In 22/31 configuration, departure aircraft on runway 31 can be airborne at the runway crossing point, requiring wake vortex separation between it and the trailing arrival. Since B757 requires larger wake vortex separation than a Large aircraft type, the tower and TRACON controllers need to coordinate and create a larger inter-arrival spacing gap reserved for the individual B757 departure. Such coordination is typically initiated between 10 to 20 minutes before takeoff, once B757 is in the taxiway queue. Tactical adjustment of the schedule due to B757 will require new operational procedures to accommodate it and stress the concept.

\section{Traffic Characteristics}

Despite a relatively small surface area with a limited number of runways and taxiways, LGA handles a large volume of traffic that normally starts around $7 \mathrm{am}$ local time and is maintained at steady, high levels until around $8 \mathrm{pm}$ in the evening. The initial traffic consists mostly of departures, but by the time the traffic nears its peak at around 10am it consists equally of departures and arrivals. The aircraft types at LGA are predominantly Larges, with a few additional Smalls and B757s throughout the day. The homogenous aircraft types simplify the inter-arrival and arrival-departure spacing requirements. Table 1 summarizes the actual arrival rate during weekday VFR operations at LGA, observed in 2013 using ASPM data. The median arrival rate varied from 32 to 35 depending on the time of the day. The mode, which indicated the arrival rate that occurred most often, showed a high arrival rate of 38 and 39 during the afternoon until the evening. 
Table 1. Summary statistics of the arrival rates at LGA in 2013

\begin{tabular}{|c|c|c|}
\hline & Median & Mode \\
\hline $\begin{array}{c}\text { Morning } \\
(6: 00-11: 59)\end{array}$ & 32 & 32 \\
\hline $\begin{array}{c}\text { Afternoon } \\
(12: 00-17: 59)\end{array}$ & 35 & 38 \\
\hline $\begin{array}{c}\text { Evening/Night } \\
(18: 00-23: 59)\end{array}$ & 32 & 39 \\
\hline
\end{tabular}

For the simulation study, the traffic demand was designed to mimic the actual arrival rates that were observed in the median and mode in Table 1. Table 2 summarizes the arrival aircraft counts there were designed into the scenarios:

Table 2. Aircraft count per hour for High and Moderate traffic scenarios

\begin{tabular}{|l|c|c|c|c|}
\hline & $\begin{array}{c}\text { From ZNY } \\
\text { (Empyr) }\end{array}$ & $\begin{array}{c}\text { From ZDC } \\
\text { (Empyr) }\end{array}$ & $\begin{array}{c}\text { From ZBW } \\
\text { (Haarp) }\end{array}$ & $\begin{array}{c}\text { Aircraft } \\
\text { Count }\end{array}$ \\
\hline High Traffic & 11 & 17 & 13 & 41 \\
\hline Moderate Traffic & 15 & 14 & 6 & 35 \\
\hline
\end{tabular}

The simulation runs lasted 80 minutes. The initial 20 minutes were populated with fewer arrivals to allow the controllers to get acclimated to the traffic scenario and therefore were excluded from the data analyses. No departures were released during this period. Therefore, the last 60 minutes were used to provide the core data for the analyses.

\section{E. Apparatus}

Multi-Aircraft Control System (MACS) software was used to emulate Standard Terminal Automation Replacement System (STARS) displays shown on large-format monitors similar to those used in current air traffic control facilities. Keyboards similar to those used in the field further helped to replicate the look and feel of these facilities. MACS software provides a high fidelity environment in which to prototype scheduling tools, to simulate the air traffic, and to collect data ${ }^{8}$. In addition to STARS displays, MACS software was used to prototype Planner, tower, and pseudo-pilot stations.

\section{F. Procedures}

1. Freeze Horizon and Traffic Delivery prior to TRACON Entry

In the study, the arrival scheduler's freeze horizon was set at $150 \mathrm{~nm}$ from the airport, which froze most of the arrivals just prior to their tops-of-descent. Although aircraft schedules and delays were determined at this point, the procedures were designed to not show this information to the En Route controllers. Instead, the En Route controllers were asked to deliver the arrivals to the meter fixes no less than 8 miles-in-trail (MIT) apart across all three conditions, similar to current operations.

Although arrivals could have been metered in the En Route sectors, it was decided to keep the operations the same as current LGA operations. Instead of metering, LGA controls the departure times of the internal departures (e.g., departures from first tier Center airports) using the scheduler. Since internal departures contribute approximately half of the traffic to LGA, controlling the departure times from these airports has been sufficient and En Route metering was not required.

\section{Runway Schedule Adjustments:}

The Planner position was envisioned to be a part of TMU and therefore was located apart from TRACON and Center control rooms in the simulation setup. The Planner station had a modified DSR (Display System Replacement) and STARS displays, set at different spatial granularities, to monitor the En Route and TRACON airspace that contained the LGA arrivals. The Planner also had a runway timeline embedded into the DSR and STARS displays that he could use to modify the runway schedules when needed. The three traffic situation displays showed LGA arrivals at different zoom levels focused on the traffic at the TRACON, Center, and the Final sector (shown left to right on Figure 6). Figure 6 also shows a small tablet on the left for the communication system and a display on the right that showed the departure queue at the airport to view the current departure demand.

7

American Institute of Aeronautics and Astronautics 


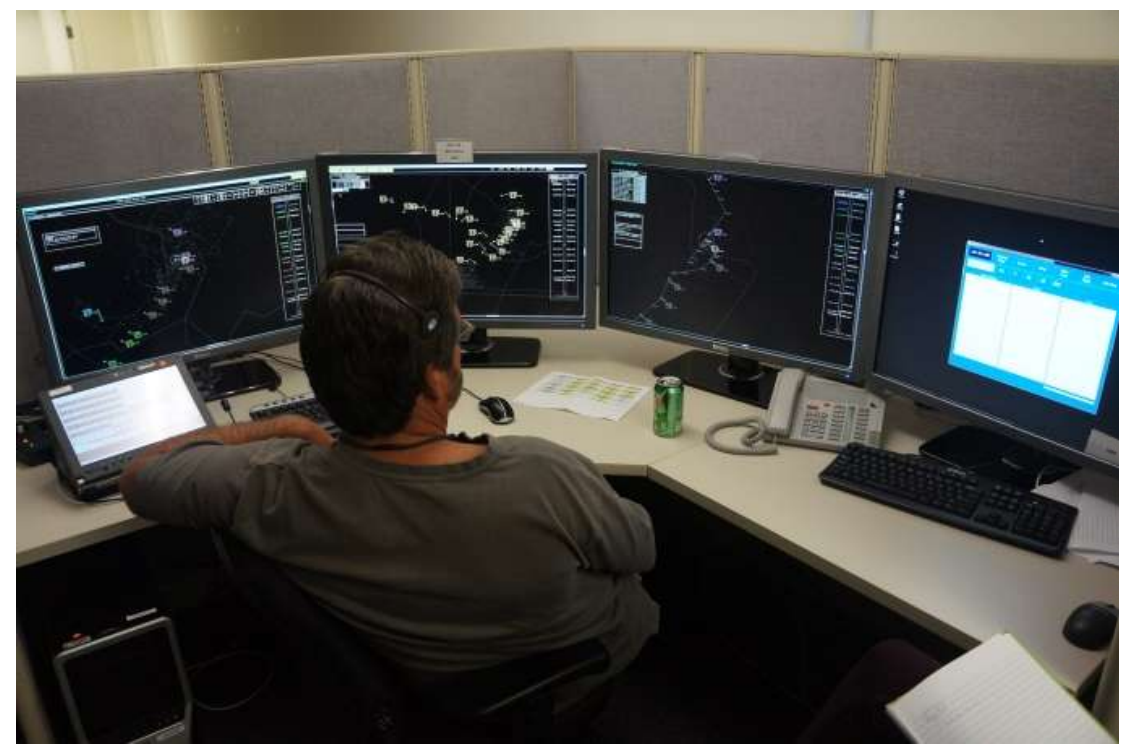

Figure 6. Planner station

In the Baseline condition, there was no scheduling task and, therefore, the Planner's involvement was limited. Communications occurred primarily between the Center and TRACON supervisors and then with controllers to determine the proper sequence of arrivals.

In the TSS condition, all arrivals had scheduled times of arrival at the runway. However, since the En Route controllers were delivering the aircraft at MIT $\geq 8$ miles, the arrival sequence sometimes differed from the TSS scheduled sequence. The Planner monitored those situations and adjusted, swapped, and/or rescheduled the STAs in ways to reduce the delays that the TRACON controllers would see at the TRACON entry point.

In the DSAS condition, the Planner was able to manipulate the schedule via a timeline interface to optimize the departure gaps. The Planner had access to a scheduling support tool via a flyout menu on the timeline that assigned a pre-determined gap size between arrivals to accommodate varying types of departures: single, double, triple, or a B757. Except for these new departure-sensitive scheduling adjustments, the procedures were the same as in the TSS condition.

Figure 7 shows our prototype interface for DSAS functionality that was available at the Planner station. The left figure shows DAL1046 and a bracket that indicates the minimum wake vortex spacing for that arrival. The green bars indicate the "slack capacity" that is in excess of the minimum inter-arrival spacing that is needed. The middle shows the options presented to the Planner when the Planner has right clicked on the call sign, which brings up the options for different types and numbers of departures (e.g., WV for normal wake vortex spacing, Double for spacing needed for two departures, Triple for three departures, etc.).
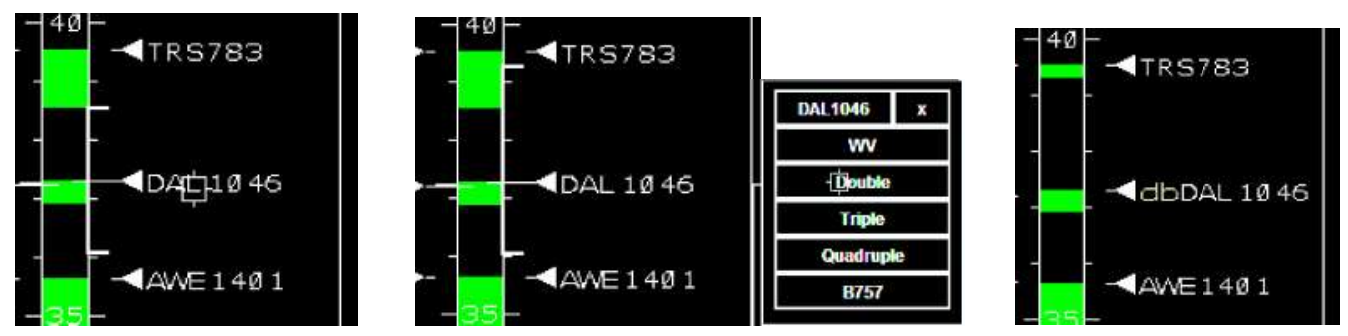

Figure 7. DSAS schedule adjustment sequence (from left to right)

When the Planner hovers over the item "Double" on the menu, the bracket for DAL1046 becomes larger to preview the spacing needed for two departures. As shown on the right figure, when the Planner assigns the "Double"

8

American Institute of Aeronautics and Astronautics 
spacing into the schedule, the word "db" for double appears next to the callsign DAL1046 to indicate that the larger spacing has been reserved for two departures and the green bar shows less slack capacity near the TRS78. The updated schedule and the "db" assignment are then propagated throughout the system to show the updated schedule to the impacted controllers. For the TRACON controllers, their STA conformance task remains the same as in TSS operations - i.e., the TSS slot markers automatically reflect the updated schedule created by the DSAS operations and the controllers conform to the STAs by putting the aircraft in their slot markers using speed and route amendments.

\section{Schedule Conformance in the TRACON}

In the Baseline Condition, there was no scheduling task. The two feeder sectors, Empyr and Haarp, sequenced their respective traffic flows and the Final sector controller merged the two flows in his sector. A Sequencer position was staffed to monitor the traffic flows from the feeder sectors. When there appeared to be a tie between two aircraft in the Final sector, the Sequencer generally coordinated between the Haarp and Final sectors to vector an aircraft in Haarp to follow behind an aircraft in Final. This was due to the lighter traffic volume and the ample maneuvering space in the Haarp sector.

The Final controller was responsible for safely and efficiently landing aircraft on 22. The Final controller maintained $2.5 \mathrm{~nm}$ spacing between arrivals for nominal VFR wake vortex separation when able, but allowed less spacing when workload was excessive or the merge became challenging. This behavior mimicked those of Final controllers in current operations.

In the TSS and DSAS conditions, controllers used TSS slot marker advisory circles to help them deliver each aircraft on its assigned STAs. Their tasks did not differ significantly for the TSS and DSAS conditions, except they saw special spacing designations whenever the Planner assigned special spacing for multiple departures (e.g., "db" and "tr" on the timelines) in the DSAS condition. In both of these conditions, the slot markers implicitly coordinated the Empyr and Haarp traffic flows, so the Final controller or the Sequencer did not need to work hard to merge the flows in the Final sector.

\section{Departure Clearance}

The tower Local controller, who was a confederate and not a test participant, issued the landing and departure clearances. The simulation platform did not have a full surface simulation capability but was able to show aircraft taking off and landing on the runways on a radar-like display. The tower Local controller also had an emulation of the Departure Spacing Program (DSP) tool that the controllers use in the field (see Figure 8).

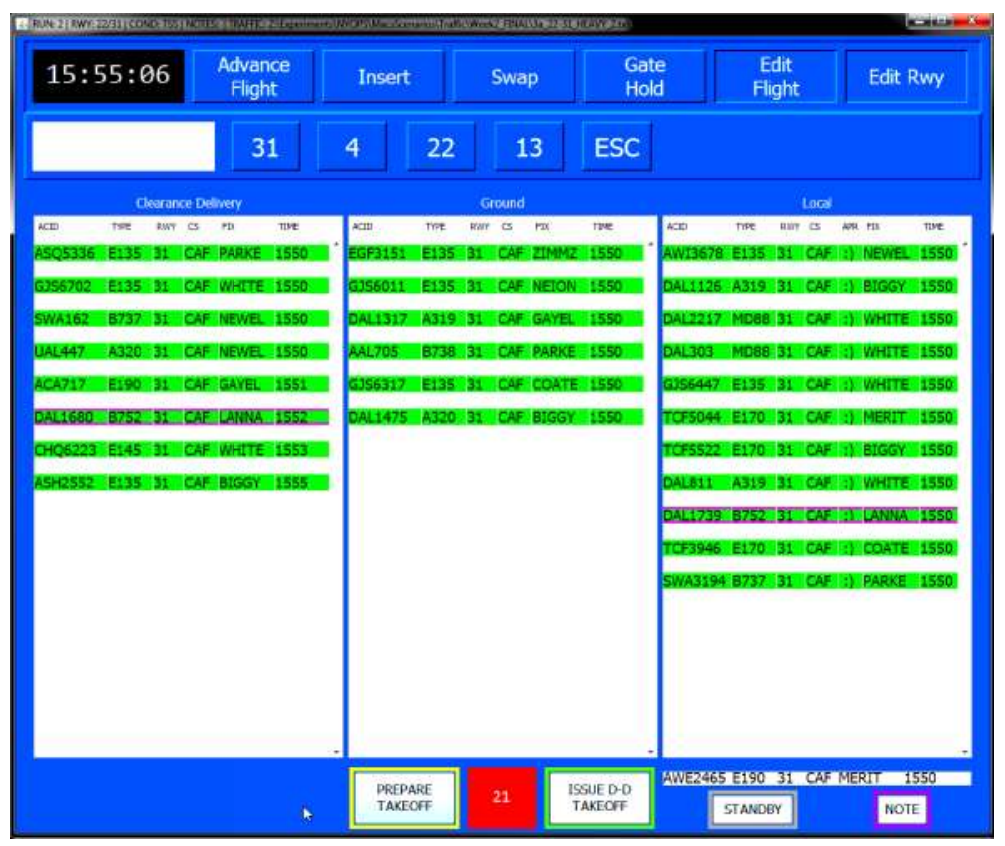

Figure 8. DSP tool emulation with custom departure clearance capability 
DSP shows the departures that are in the queue for the Clearance Delivery, the Ground controller, and the Local controller. This tool allowed the tower Local controller to view the departure queue on the taxiway (in the right column) and to put an aircraft on standby if needed (e.g., to hold a B757 departure to wait for its gap). In addition to the normal DSP functionality, a departure clearance capability was added to release the departures into the simulation environment.

Based on an LGA SME's input, the tower Local controller was given a clear set of guidelines on when he could issue the departure clearance. These were:

- Clear the departure after the arrival passes the runway crossing point. (The controller pressed the "prepare take-off" button when the arrival passed its runway threshold and the DSP tool estimated the time it took for the arrival to reach the crossing point before releasing the departure. This also allowed the controller to cancel the clearance if needed.)

- Do not clear the departure if the trailing arrival is within $2 \mathrm{~nm}$ of its runway threshold.

- When multiple departures are cleared, wait at least 45 seconds after the lead departure before clearing the trailing departures. (The 45 seconds was deemed sufficient spacing in VFR conditions based on the SME's input.)

- A B757 departure can be cleared if the trailing arrival is at least $6 \mathrm{~nm}$ from the runway threshold.

- Due to wake vortex spacing requirements, wait 94 seconds after the B757 departure to clear a second departure. (This time would allow the preceding 757 departure to be at least 1.5 miles off the end of the runway before the launching of the second departure).

At the beginning of each simulation run, the tower Local controller did not clear any departures until 20 minutes into the scenario. At 20 minutes, the DSP emulation tool was initiated with 10 departures in the Local queue and subsequently moved a departure into the queue every 75 seconds. The DSP tool recorded the number of departures cleared for take-off, the number of departures left in the queue, and the time that the departures spent in the queue.

\section{Tactical Schedule Adjustments for B757 Departures in 22|31 Runway Configuration}

In the nominal DSAS operations, the Planner can modify the schedule prior to controller intervention, resulting in minimal coordination and impact on controller workload. However, in situations where tactical schedule adjustment is needed after the controllers have begun to take actions, more coordination is required. One of those cases that occurs regularly is B757 departures in the 22|31 configuration. At LGA, the 22|31 configuration requires a special inter-arrival spacing when a B757 departs on runway 31 because the departure becomes airborne at the crossing point, needing a four mile wake vortex spacing between the B757 departure and the trailing arrival.

In current operations, when a B757 departure is at least seventh in the departure queue, the tower cab coordinator notifies the TRACON (either the Final controller, supervisor, or a "Sequencer" who monitors/coordinates the arrival sequence, depending on who is available). The Sequencer (or the Final controller if the Sequencer is not available) assesses the arrival traffic and timeline to determine which point within the arrival stream would be the best to build the necessary gap to allow for a B757 departure. The Sequencer checks with the affected feeder controller to see if the gap would be possible to make and that it would not interfere with anything else at the time. Once the appropriate arrival pair is identified for the gap, the Sequencer coordinates with the tower to specify the location of the gap. In the 22/31 configuration, the tower Local controller has access to an alternate taxiway where B757s can be taken out-of-sequence and held for up to approximately 10 minutes to wait the for the proper gap without being completely out of the departure queue.

In the TSS and DSAS operations, the Sequencer also calls the Planner so that the Planner can adjust the TSS/DSAS STAs to match the gap that the TRACON controllers are building. Whenever the Planner adjusts the STAs, the slot markers "jump" to their new locations. With proper coordination, the TRACON controllers are aware of the change and are already moving the aircraft to the new locations. The Planner adjusts the schedule similarly in TSS and DSAS conditions, but DSAS has a couple of advantages. First, the DSAS tool has a special B757 spacing option in the fly-out menu that the Planner can use to assign the spacing. The assignment is then propagated across the system. Secondly, the Planner might have already created a double or a triple gap for multiple departures in the vicinity of where the B757 gap is needed. In that situation, the Planner can "re-purpose" the gap, which results in less controller workload due to similar gap sizes. 


\section{Participant Feedback}

During the simulation runs, the controllers were prompted every three minutes to report their current workload on a scale of 1 to 6 using Workload Assessment Keypads (WAKs). Ratings of 1 and 2 were considered to be low workload, ratings of 3 and 4 were considered to be medium workload, and ratings of 5 and 6 were considered to be high workload.

After each run, the controllers responded to an online post-run survey, and after the simulation, they responded to a post-sim survey and participated in a debrief discussion. Survey questions included those on workload, acceptability, feasibility and safety of the operations and coordination. The questions were typically binary (yes/no), or involved ratings on a 5-point Likert scale, ranging from 1 (lowest) to 5 (highest). Space was made available for comments on both survey instruments. WAK and post-run data were analyzed with repeated measures Analyses of Variance (ANOVAs). Where error bars are depicted in figures, they are $95 \%$ confidence intervals (CIs) adjusted for repeated measures analysis of variance per Morey ${ }^{9,10}$ and Baguley ${ }^{11}$ unless otherwise noted.

\section{Results}

The overall results showed that during high arrival demand period, departure throughput could be increased under TSS and DSAS operations without compromising the arrival throughput when compared to Baseline. The details of the DSAS concept, operational procedures, and summary results were published in ATM $2015^{1}$ and the authors encourage the readers to refer to that paper for step-by-step description of the operational concept and procedures. This paper provides complementary analyses that expand on the detailed results from the study with respect to throughput, conformance, safety, flight time/distance, etc., which were omitted in the earlier paper.

\section{A. Throughput}

The arrival and departure throughput was measured by counting the number of aircraft landed and departed per hour. Figure 9 summarizes the arrival and departure rates across the six conditions. In the High traffic condition, the arrival rate was similar across the three experimental conditions $(N=41,41$, and 39 arrivals per hour for Baseline, TSS, and DSAS, respectively). These arrival rates were designed to be similar to the high arrival demand observed most frequently during busy afternoons and evenings in the operational data (see the mode column in Table 1). During the High traffic condition, the departure rate/throughput was increased from 38 / hour (Baseline), to 44 / hour (TSS) to 47 / hour (DSAS). In the Moderate traffic condition, the arrival rate was closer to the median rate in Table $1(N=35,35$, and 34 arrivals per hour for Baseline, TSS, and DSAS, respectively). In this condition, DSAS improved the departure throughput compared to the other two conditions, but TSS did not show significant improvement over Baseline ( $N=45,46$, and 50, for Baseline, TSS, and DSAS, respectively).

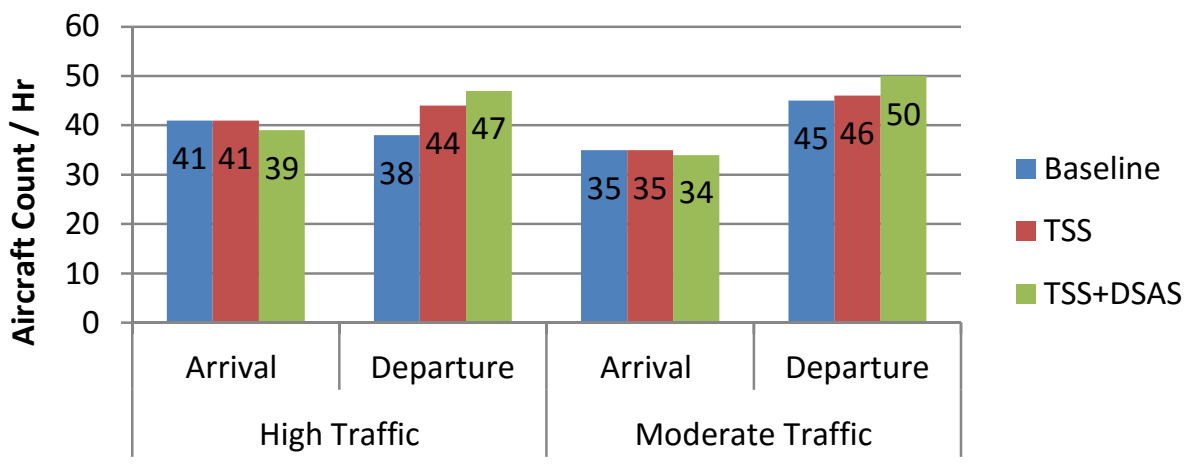

Figure 9. Count of arrivals and departures per hour by traffic level and operational condition

\section{B. Impact of Missed and Extra Departures on the Departure Throughput}

One-for-one departure/arrival operation is the goal at a when the arrival demand is high, but it is very difficult to control the inter-arrival spacing to preserve the departure slots in current operations. The following section examines how missed departure slots and extra departure gaps in each condition contributed to the differences in the departure throughput.

11

American Institute of Aeronautics and Astronautics 


\section{Missed Departures}

One hypothesis why TSS condition can improve the departure throughput compared to Baseline is that managing an arrival schedule to the runway threshold ensures consistent inter-arrival spacing to the runway, thereby implicitly preserving the departure slots if the controllers can deliver the arrivals on schedule. If TSS operation could reduce the number of inter-arrival spacing that were too tight to fit a departure, it should result in higher departure throughput compared to Baseline which did not require inter-arrival spacing to preserve a departure slot. The potential for missed departure slot due to tight inter-arrival spacing was more likely in High traffic since the higher density of arrival traffic forced the Final controller to deliver arrivals on minimum wake vortex spacing than in Moderate traffic which has natural slack in the spacing to allow greater buffer between arrivals.

Table 3 summarizes the number of missed departure slots across traffic levels and operational conditions, represented by the number of inter-arrival spacing that the tower Local controller deemed too tight to release a departure aircraft. In the High traffic condition, the number of missed departure slots were highest in Baseline $(N=$ 9) compared to TSS and DSAS ( $N=1$ and 0 , respectively).

Table 3. Number of missed departure slots

\begin{tabular}{|c|c|c|c|}
\hline & Baseline & TSS & DSAS \\
\hline High Traffic & 9 & 1 & 0 \\
\hline Moderate Traffic & 4 & 4 & 4 \\
\hline
\end{tabular}

In the Moderate traffic condition, there were no difference across conditions $(N=4,4,4$ for Baseline, TSS, and DSAS, respectively), suggesting that explicit scheduling of the inter-arrival spacing did not improve ensuring the departure slots. A closer look at the circumstances that resulted in four missed slots per condition suggested that even with TSS and DSAS scheduling, there were instances when the controllers sequenced the arrivals closer than scheduled, resulting in inter-arrival spacing around $2 \mathrm{~nm}$, which was not enough spacing for a departure release. If the TSS / DSAS schedule in the simulation were adjusted with larger spacing buffers that would correspond to the lower arrival demand in moderate traffic, then there should have been less occurrence of missed departure slots in those conditions, even when the arrivals are slightly out-of-conformance from the schedule.

\section{Extra Departures}

Although the increase in departure throughput could be partially attributed to a reduction in missed departure slots, tight inter-arrival spacing that resulted in missed slots might also create excess spacing in the subsequent arrival pairs such that extra departures (e.g. 2 departure between 1 arrival pair, 3-for-1 departures/arrival pair, etc.) between subsequent arrivals could potentially minimize the negative impact due to the missed departure slots. Therefore, the number of extra departures between arrival pairs was also recorded. Extra departures between arrival pairs required 39 seconds spacing behind another departure which was a Large aircraft type and 94 seconds after a B757 departure. In the Moderate traffic condition, there were sufficient gaps in the arrival spacing to allow extra departures across all conditions. In the High traffic condition there was not much room for any extra departure gaps.

Table 4 summarizes the number of missed departure slots between arrival pairs, number of extra departures released above the one-for-one arrival/departure operations, and the net difference in the extra departures released per condition after subtracting the missed departure slots. The results show that in the High traffic condition, the improvement in the departure throughput was mainly due to having fewer missed departure slots in TSS and DSAS operations while in the Moderate traffic condition, the improved departure throughput was mainly due to the ability to creating optimal arrival spacing in DSAS that improved the number of extra departures that could be released.

Table 4. Net amount of extra departures released above one-for-one arrival/departure operations

\begin{tabular}{|c|c|c|c|c|}
\hline & & Baseline & TSS & DSAS \\
\hline \multirow{3}{*}{ High Traffic } & Missed Departure Slot & 9 & 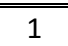 & 0 \\
\hline & Number of Extra Departures Released & 6 & 5 & 7 \\
\hline & $\begin{array}{l}\text { Net Difference of Extra Departures } \\
\text { accounting for Missed Departures }\end{array}$ & -3 & 4 & 7 \\
\hline \multirow{3}{*}{ Moderate Traffic } & Missed Departure Slot & 4 & 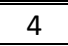 & 4 \\
\hline & Number of Extra Departures Released & 14 & 15 & 19 \\
\hline & $\begin{array}{l}\text { Net Difference of Extra Departures } \\
\text { accounting for Missed Departures }\end{array}$ & 10 & 11 & 15 \\
\hline
\end{tabular}

12

American Institute of Aeronautics and Astronautics 
Table 5 elaborates on the number and types of extra departure releases by conditions. In the High traffic condition, most of the extra departures were released as a 2-for-1 (two departures per inter-arrival spacing) for all three conditions. For the Moderate traffic condition, there were increased number extra departure slots in the DSAS condition, likely due to extra departure slots created when DSAS operations and the less-than-full arrival schedule allow the arrival schedule to be adjusted to create extra departure spacing.

Table 5. Number and types of extra departure releases between arrival pairs

\begin{tabular}{|c|c|c|c|c||}
\hline & Departures per Arrival Pairs & Baseline & TSS & DSAS \\
\hline \hline \multirow{4}{*}{ High Traffic } & 2-for-1 (Double) & 6 & 5 & 5 \\
\cline { 2 - 5 } & 3-for-1 (Triple) & 0 & 0 & 1 \\
\cline { 2 - 5 } & 4-for-1 (Quadruple) & 0 & 0 & 0 \\
\cline { 2 - 5 } & 5-for-1 & 0 & 0 & 0 \\
\hline \hline \multirow{3}{*}{ Moderate Traffic } & 6-for-1 & 6 & 3 & 0 \\
\cline { 2 - 5 } & 2-for-1 (Double) & 1 & 2 & 1 \\
\cline { 2 - 5 } & 3-for-1 (Triple) & 0 & 0 & 3 \\
\cline { 2 - 5 } & 4-for-1 (Quadruple) & 2 & 1 \\
\cline { 2 - 5 } & 5-for-1 & 0 & 0 \\
\hline \hline
\end{tabular}

\section{Delay}

Another method of examining the departure benefits was to calculate the taxi-out delays. In each simulation run, departures were added every 75 seconds (i.e., a rate of 48 aircraft per hour). During the simulation runs, the queue length increased gradually over the first 40 minutes of departure operations and stabilized during the last 20 minutes. Averaging the time in the queue during the last 20 minutes of the simulation, the time in the queue was reduced from 23.7 min. per aircraft in Baseline to $17.2 \mathrm{~min}$. in TSS and $13.3 \mathrm{~min}$. in DSAS during the High Traffic scenarios (Table 6).

Table 6. Departure time in queue and taxi-out delay savings (min.) during the last 20 min of the simulation

\begin{tabular}{|c|c|c|c|c|}
\hline & & Baseline & TSS & DSAS \\
\hline \hline \multirow{3}{*}{ High Traffic } & Time in Queue & 23.7 & 17.2 & 13.3 \\
\cline { 2 - 5 } & Delay Savings & - & $\mathbf{6 . 5}$ & $\mathbf{1 0 . 4}$ \\
\hline \hline \multirow{2}{*}{ Moderate Traffic } & Time in Queue & 17.4 & 16.5 & 11.9 \\
\cline { 2 - 5 } & Delay Savings & - & $\mathbf{0 . 9}$ & $\mathbf{5 . 5}$ \\
\hline
\end{tabular}

The delay savings were calculated by subtracting the TSS or DSAS time from the Baseline time. The results showed that departures in the TSS and DSAS conditions had an average of 6.5 and 10.4 minutes less taxi-out time respectively compared to the Baseline conditions. In Moderate Traffic, the TSS condition produced little delay savings ( 0.9 minutes) while DSAS condition reduced the delays by 5.5 minutes, mirroring the benefit characteristics found in the throughput data.

\section{Inter-arrival Spacing}

To investigate how well TSS and DSAS operations optimized the inter-arrival spacing for departure throughput, the inter-arrival spacing of aircraft landing in the 22|31 configuration was examined. The spacing values were calculated as the difference in arrival times between successive arrivals at the runway threshold.

Figure 10 illustrates the inter-arrival spacing data taken during the simulation runs across the conditions. The spacing data in the Baseline condition shows how the spacing values were distributed between 60 and 120 seconds, with few outliers with larger spacing. (In the Moderate traffic scenario, there are natural gaps in the arrival schedule which resulted in larger spacing). Spacing values from 60 to 75 seconds roughly translates to the minimum allowable $2 \mathrm{~nm}$ of spacing to $2.5 \mathrm{~nm}$ nominal wake vortex spacing at the runway threshold in VFR. Spacing values below 60 seconds (shown in red) resulted in missed departure slots due to insufficient spacing.

13

American Institute of Aeronautics and Astronautics 


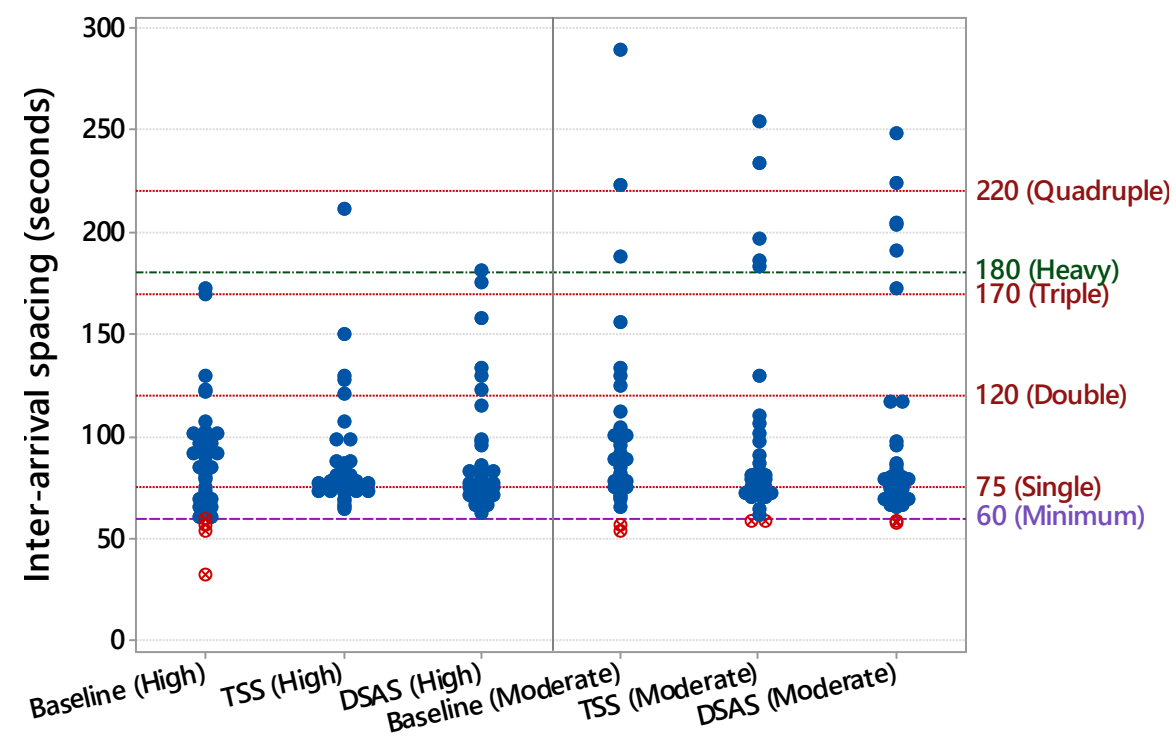

Figure 10. Inter-arrival spacing across conditions. The dotted lines indicate the different inter-arrival spacing parameters in the TSS and DSAS schedulers

In the Moderate traffic condition, 34 arrivals common across Baseline, TSS, and DSAS were analyzed. Out of the 34 arrivals in Baseline, six were delivered with spacing between 60 and 75 seconds, and two were delivered with less than 60 seconds spacing with the trailing arrival with the closest pairing separated by 53 seconds at the runway threshold. In the TSS condition, 11 were delivered with less than 75 seconds and two were delivered at 58 seconds. Compared to Baseline, the TSS condition resulted in a tighter clustering of spacing values around 75 seconds, where the TSS scheduler set the wake vortex spacing. Similarly, DSAS operations also resulted in most spacing values around 75 seconds and very few below 60 seconds. Results for the DSAS condition were very similar to those for TSS albeit with a slightly wider distribution of spacing values. In the DSAS condition, 11 were delivered with less than 75 seconds spacing and two were delivered with less than 60 seconds spacing, at 57 and 58 seconds of spacing ${ }^{6}$.

In the High traffic condition, 39 arrivals common across Baseline, TSS, and DSAS were analyzed. In the High Traffic condition, Inter-arrival spacing in the High traffic condition showed greater differences between conditions particularly with respect to Baseline. Out of the 39 arrivals, 14 were delivered with less than 75 seconds spacing, and 4 were delivered with less than 60 seconds spacing (the minimum spacing recorded was 32 seconds). The TSS condition had perhaps the tightest clustering of inter-arrival spacing centered at the wake vortex value of 75 seconds. A total of 12 were delivered with less than 75 seconds spacing but greater than 60 seconds. No aircraft were delivered with less than 60 seconds spacing. The DSAS condition had results similar to those observed in the TSS condition with a similar distribution. Thirteen arrivals delivered with less than 75 but greater than 60 seconds spacing. No aircraft were delivered with less than 60 seconds spacing just as in the TSS condition. DSAS spacing also seems to be clustered around double and triple departure spacing times, probably due to the Planner's assignment of double and triple departure spacing.

Figure 11 illustrates the spacing distribution as a time series data. After an initial variability in spacing at the start of the simulation run, tighter spacing around 75 seconds spacing for one-for-one operations are visible for TSS and DSAS operations under the High traffic condition. A similar pattern was not visible in the Moderate traffic condition.

${ }^{6}$ The criterion of 60 seconds or less spacing as missed departure slots, which was derived from timing of arrivals and departures in the operational data, resulted in fewer count of misses than the actual count in Table 3, which were based on the tower Local controller's decision to not release the departures when he deemed the interarrival spacing to be too tight. The discrepancy suggests that the heuristics that were used in the simulation based on SME inputs and the sampled data mismatched slightly but the overall trends remain the same. 


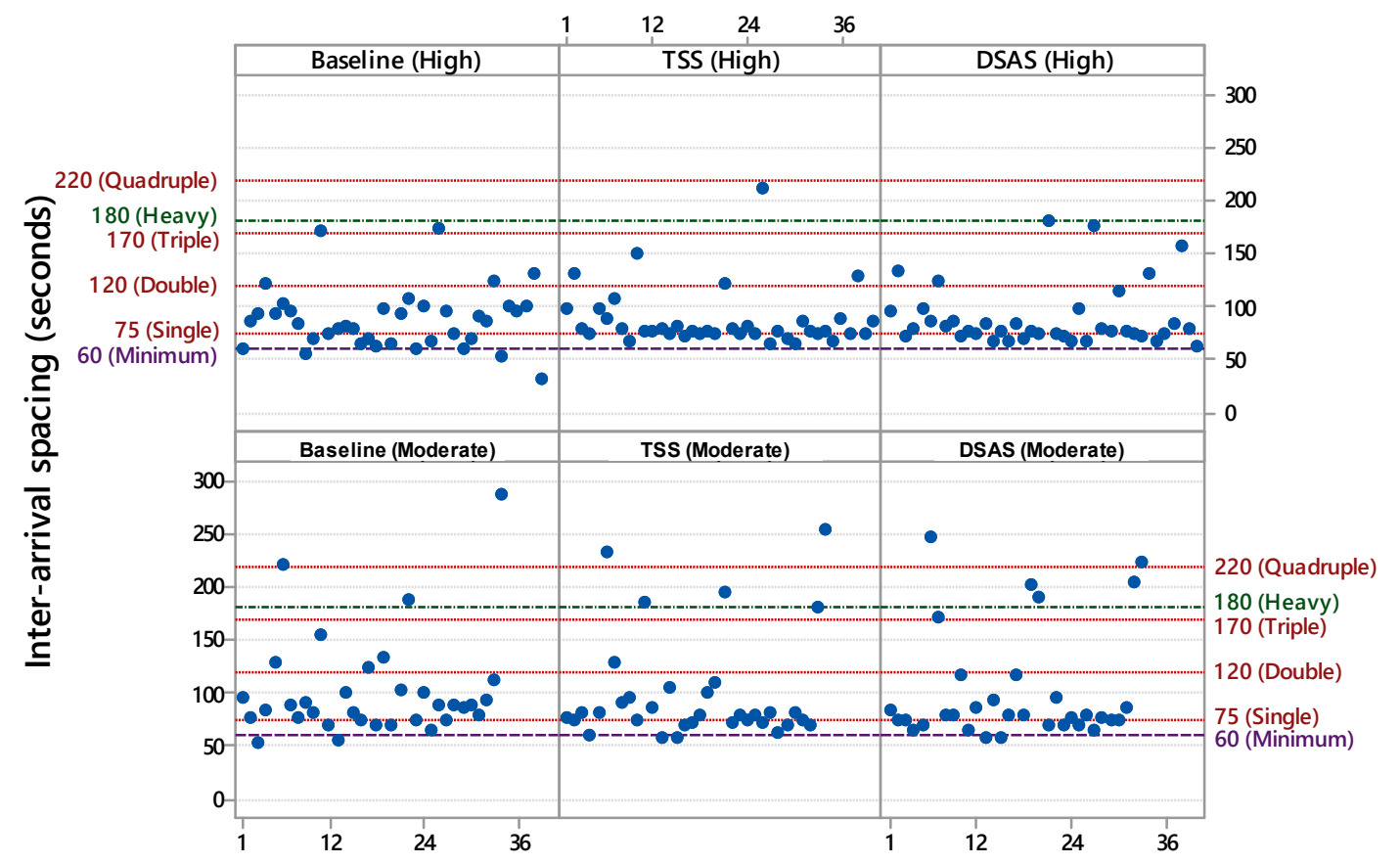

Figure 11. Inter-arrival spacing (seconds) shown as a time series data along the simulation run time

To further analyze inter-arrival spacing results, box and whisker plots were generated for each traffic level and condition within and tests of variance were conducted for comparison. The upper and lower extents (or hinges) of the boxes in Figure 12 represent the $75^{\text {th }}$ and $25^{\text {th }}$ quartiles respectively with the median represented by the horizontal line within the box. The whiskers extend to $1.5 \mathrm{x}$ the Interquartile Range (IQR). The dots above the whiskers are considered outliers and represent data points that exceed the extent of the whiskers. These points, however, are not necessarily outliers in the traditional sense since extra spacing was intentionally applied to allow for the release of additional departures.
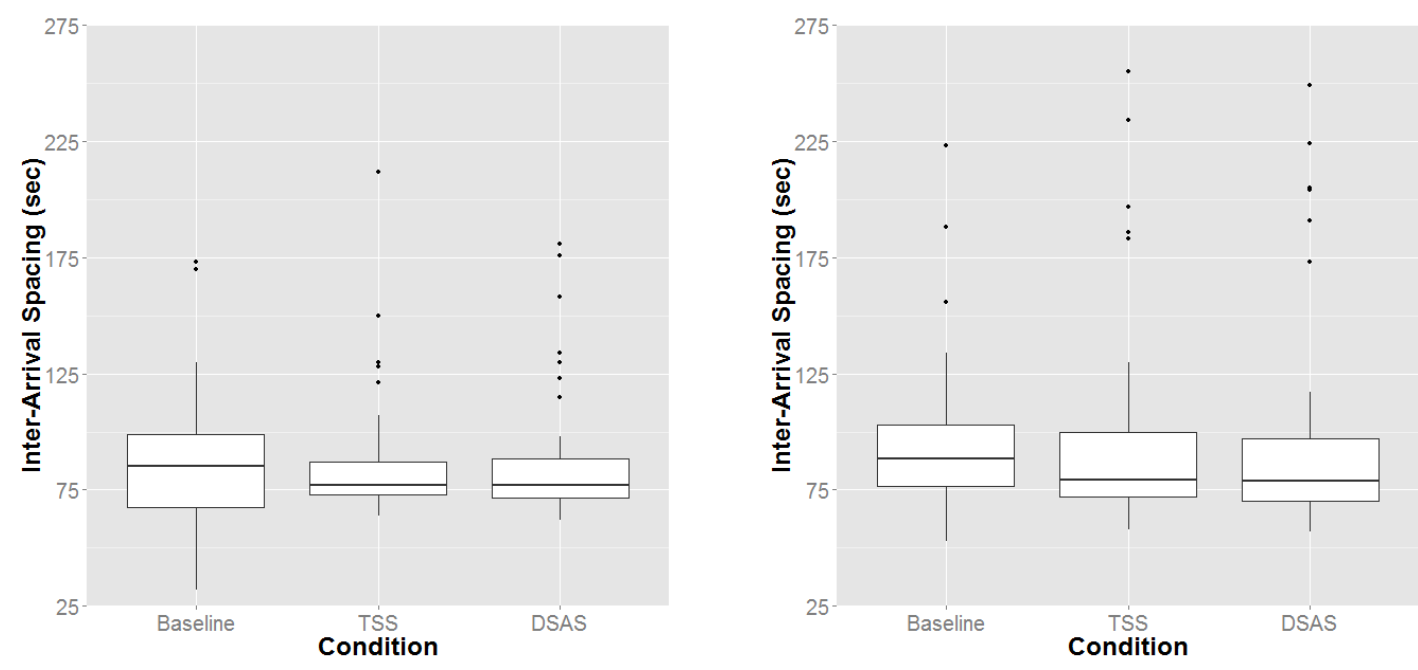

Figure 12. Box and whisker plots for the High (left) and Moderate traffic conditions (right) 15

American Institute of Aeronautics and Astronautics 
The left subplot of Figure 12 presents the box and whisker plot for the High traffic condition where it can be seen that in this case, inter-arrival spacing in the Baseline condition was noticeably more varied with a higher median and hinges that extend further than the other conditions'. Despite these differences, however, a Levene's test for equality of variances did not find a significant difference in inter-arrival spacing variance between the conditions, $F(2,116)=.69, p=.50$. The right subplot of Figure 12 presents the box and whisker plot for the Moderate traffic condition where it is shown that the results for each condition were quite similar. Although the Baseline condition resulted in a slightly higher median inter-arrival spacing value and the TSS condition showed slightly more variance, a Levene's test for equality of variances did not find a significant difference in inter-arrival spacing variance between the conditions, $F(2,98)=.07, p=.94$.

Further analysis on DSAS operations were done to examine on how well DSAS operations created optimal inter-arrival spacing for departures. Figure 13 plots the average inter-arrival spacing for the different number of departures between an arrival pair (excluding the B757s), as well as average spacing for the missed departure slot. For Moderate and High traffic, the one-for-one operations averaged 78.1 and 79.1 seconds spacing which was close to the 75 seconds wake vortex spacing used by the TSS scheduler for the Large aircraft types. However, the 2-for-1 operations resulted in 152.8 and 144.8 seconds spacing for Moderate and High traffic, respectively, which is much higher than the 120 seconds target spacing. The large average spacing values for 2 -for-1 seems to be a result of two values in each traffic level that ranged from 150 to 200 seconds, which in turn biased the average. The spacing for 3for-1 or greater varied around target spacing but there were too few data points to draw conclusions.
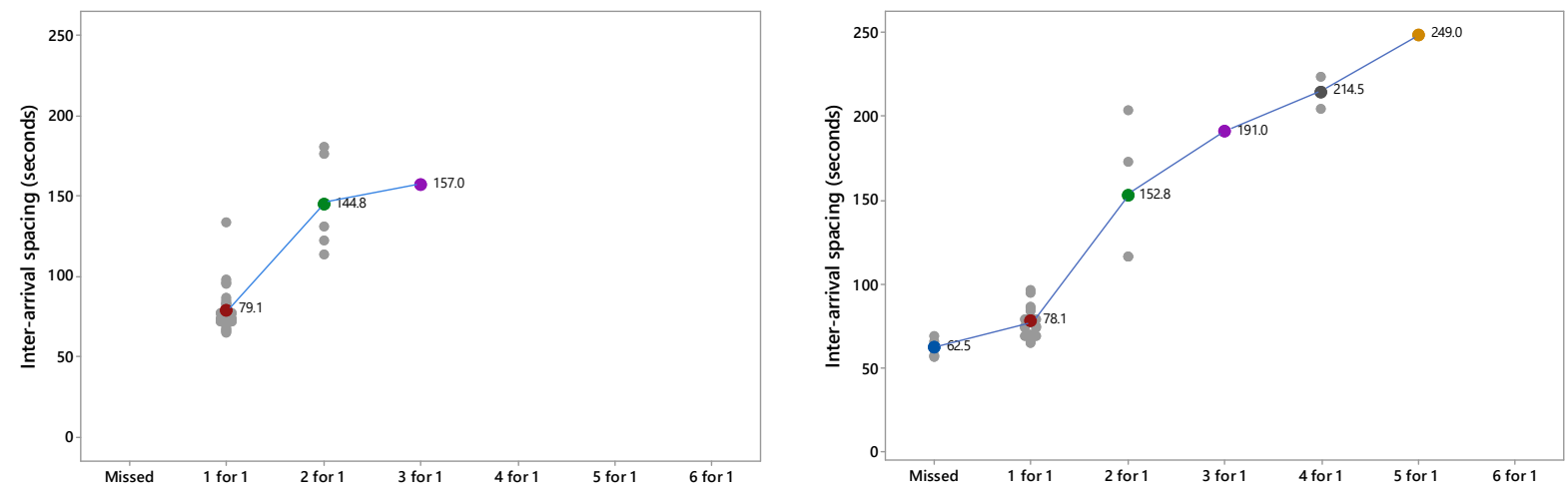

Figure 13. Inter-arrival spacing for $n(=0,1,2,3,4$, and 5) departures in High (left) and Moderate traffic conditions (right) under DSAS operations

Figure 14 shows time series plots of the variability to the target spacing by subtracting the assigned from the actual spacing. As shown in the figure, one-for-one spacing has a distribution that is slightly biased towards greater actual spacing than the assigned one. The number of data samples for the spacing greater than one-for-one is sparse and therefore is hard to draw any conclusions, but the figure shows a couple of data samples in 2 -for 1 case in both Moderate and High traffic conditions that resulted in spacing values that could have allowed an extra departure. Further examination is needed to determine the context in which only two departures were released in a spacing that could have allowed three.
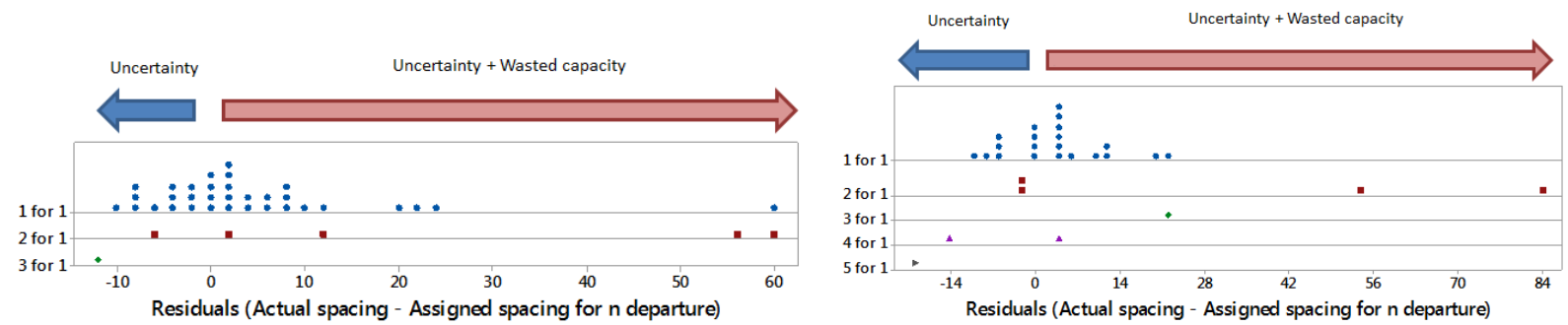

Figure 14. Residuals for $n(=1,2,3,4$, and 5) departure in High (left) and Moderate traffic condition (right) under DSAS operations

16

American Institute of Aeronautics and Astronautics 


\section{E. Tactical Adjustment of DSAS Schedule for B757 Departures}

Tactical schedule adjustment for B757 departures required significantly more coordination between the tower cab coordinator, TRACON Sequencer, Planner, and the controllers, creating a challenge to the DSAS operations and testing its feasibility. The overall design of the concept tried to minimize the coordination required between the tower cab coordinator, Sequencer, Planner, and the controllers by 1) requiring the Planner to adjust the schedule in the En Route airspace before the controllers were managing to the schedule, 2) having En Route controllers deliver the aircraft using MITs so that Planner's adjustments of the schedule did not impact them, , and 3) displaying and propagating the Planner assigned spacing for multiple departures automatically to the TRACON controller stations so that they understood which gaps were built for multiple departures without explicit coordination.

However, a tactical schedule adjustment for the B757 departures occurred very close to the runway threshold (somewhere around the Final sector entry), which required the tower cab coordinator to notify the Sequencer who then coordinated with the controllers to create the gap for the B757 departure. The Sequencer also needed to call and coordinate with the Planner so that he could adjust the TSS/DSAS STAs to match the gap that the TRACON controllers were building. These steps were similar to those in current operations, except that the presence of TSS scheduler required the Planner to adjust and update the schedule to reflect any tactical changes and for the controllers to manage to a new schedule for all arrivals that were impacted by the modified schedule, creating extra workload. Therefore, the details of the B757 departure procedure were examined to assess its impact on the feasibility of DSAS operation.

The tower cab coordinator was responsible for the verbal coordination with the TRACON to get the gap required for a B757 departure. He called the TRACON Sequencer to coordinate the departure gap somewhere between $7^{\text {th }}$ to $9^{\text {th }}$ in the queue. Table 7 shows the average time taken from when the coordination was initiated to the B757 departure time, as well as the average time from when the B757 enters the DSP local queue to its takeoff time as a representation of the time spent on the taxiway.

Table 7. Average time duration for coordination and taxiway occupancy for B757 departures

\begin{tabular}{||c|c|c|c|c||}
\hline & & Baseline & TSS & DSAS \\
\hline \hline \multirow{3}{*}{ High Traffic } & $\begin{array}{c}\text { Avg. Time between Coordination and } \\
\text { B757 Departure Takeoff }\end{array}$ & $12: 05$ & $10: 46$ & $19: 08$ \\
\cline { 2 - 5 } & $\begin{array}{c}\text { Avg. Time on the Taxiway Queue for the } \\
\text { B757 Departure prior to Takeoff }\end{array}$ & $15: 41$ & $14: 38$ & $22: 20$ \\
\hline \hline \multirow{3}{*}{ Moderate Traffic } & $\begin{array}{c}\text { Avg. Time between Coordination and } \\
\text { B757 Departure Takeoff }\end{array}$ & $10: 19$ & $10: 37$ & $07: 37$ \\
\cline { 2 - 5 } & $\begin{array}{c}\text { Avg. Time on the Taxiway Queue for the } \\
\text { B757 Departure prior to Takeoff }\end{array}$ & $15: 20$ & $15: 05$ & $09: 57$ \\
\hline
\end{tabular}

Overall, the average time that B757 spent on the taxiway was similar for Baseline and TSS conditions for both High and Moderate traffic conditions, although TSS condition took slightly less time than Baseline. The average time from the coordination initiation to takeoff was also similar, ranging from approximately 10 to 12 minutes. In contrast, the DSAS condition resulted in times that were considerably longer for the High and Moderate traffic conditions. In the High traffic condition, it took a long time for the TRACON Sequencer to call back the cab coordinator when the coordination was initiated. In addition, the departure slot for the B757 was really far behind its nominal departure time, requiring the B757 to be on standby to wait for its gap.

The reason for the delayed slot was that in the subsequent coordination between the Sequencer and the Planner to determine the slot for the B757, the Planner had no natural gap available in an arrival stream that was otherwise nicely spaced flow. The tightly spaced arrival stream was no accident in the DSAS condition since the Planner actively adjusts the arrival schedule to minimize any excess slack in the schedule, which may or may not put the multiple departure gap, which could be "re-purposed" for the B757 departure, in an ideal location to be used for the B757. In hind sight the cab coordinator said he should have pushed back and insisted on a closer slot.

For the Moderate traffic condition, DSAS operation had a natural gap near but slightly ahead of the B757 departure time, The natural gap for the B757 departure appeared two slots ahead of B757's expected departure time,

17

American Institute of Aeronautics and Astronautics 
thereby expediting its departure ahead of other aircraft to fit into the slot. Table 8 illustrates this point. In the High traffic condition, Baseline and TSS conditions moved B757 ahead of other departures which were put on standby to move the B757 ahead while in the DSAS condition, the B757 was put on standby while thirteen other departures moved ahead of it in the queue. In contrast, the DSAS condition under Moderate traffic required no standbys for the B757 and put two other departures on standby.

Table 8. Total count of B757 and other nearby departures on standby during B757 departure operation (top) and the average time duration for coordination / taxiway queue for each condition (bottom)

\begin{tabular}{||c|c|c|c|c||}
\hline \hline & & Baseline & TSS & DSAS \\
\hline \hline \multirow{2}{*}{ High Traffic } & Total Count of B757 on Standby & 0 & 1 & 13 \\
\cline { 2 - 5 } & Total Count of Other A/Cs on Standby & 4 & 2 & 0 \\
\hline \hline \multirow{2}{*}{ Moderate Traffic } & Total Count of B757 on Standby & 2 & 3 & 0 \\
\cline { 2 - 5 } & Total Count of Other A/Cs on Standby & 1 & 3 & 2 \\
\hline
\end{tabular}

\begin{tabular}{|c|c|c|c|c|}
\hline $\begin{array}{l}\text { Put on } \\
\text { Standby }\end{array}$ & Number of Events $(n=12)$ & $\begin{array}{c}\text { Total Number of Impacted } \\
\text { ACs }\end{array}$ & $\begin{array}{l}\text { Avg. Time } \\
\text { Coord to } \\
\text { Takeoff }\end{array}$ & $\begin{array}{l}\text { Avg. Time } \\
\text { Taxiway } \\
\text { Queue }\end{array}$ \\
\hline \multirow{2}{*}{ B757 } & $\begin{array}{c}2 \text { Moderate Traffic } \\
\text { (1 Baseline and } 1 \text { TSS) }\end{array}$ & 5 aircraft passed B757 & \multirow{2}{*}{$15: 00$} & \multirow{2}{*}{$18: 39$} \\
\hline & $\begin{array}{c}3 \text { High Traffic } \\
\text { (1 TSS and } 2 \text { DSAS) }\end{array}$ & 14 aircraft passed B757 & & \\
\hline \multirow{2}{*}{ Other AC } & $\begin{array}{c}3 \text { Moderate Traffic } \\
\text { (1 Baseline, } 1 \text { TSS, and } \\
1 \text { DSAS) }\end{array}$ & $\begin{array}{l}6 \text { ACs had to be put on } \\
\text { standby for B757s to } \\
\text { depart in front of them }\end{array}$ & \multirow{2}{*}{$10: 03$} & \multirow{2}{*}{$13: 52$} \\
\hline & $\begin{array}{c}3 \text { High Traffic } \\
\text { (2 Baseline and 1TSS) }\end{array}$ & $\begin{array}{l}6 \text { ACs had to be put on } \\
\text { standby for B757s to } \\
\text { depart in front of them }\end{array}$ & & \\
\hline Nominal & $\begin{array}{c}1 \text { Moderate Traffic } \\
\text { (1 DSAS) }\end{array}$ & Exact Placement & 05:41 & 09:38 \\
\hline
\end{tabular}

Some of the departure gaps that were created for the B757 were bigger than the 6 mile minimum required and the tower was able to get some extra departures out after the B757 departure. In fact, 9 of the 12 B757 gaps allowed for double departures (2-for-1). Baseline had 1 double in Moderate and 2 doubles in High traffic. TSS had 1 double in Moderate and 1 in High traffic. DSAS being the most precisely scheduled allowed for a double after every B757 departure (4). This is an indication that our time for B757 were too big for one-for-one operation that was targeted.

The coordination procedures to make space for the $757 \mathrm{~s}$ went very smoothly according to all participants (average rating of 4.7 from the Planner, the Sequencer, and the En Route FLM on "How well do you think the coordination for building a 757 gap worked?") The Planner commented that the coordinating between tower cab coordinator and Sequencer before involving the Planner was a "much better operation with the Sequencer and the controllers making the decision and me just implementing." The Sequencer described how the procedure worked in the DSAS condition in the post-sim survey:

"It made it easy for the TRACON controllers to have the gap [created] through the TRACON Sequencer. We would discuss the gap, start slowing or vectoring to create the gap, and then call the Planner. This made the ripple less confusing and [reduced] vectoring to get back to [the] slot markers [to keep] the pattern nice and tight."

The coordination between the TRACON controllers and the Sequencer also went smoothly. The controllers were asked whether the coordination was initiated by them or by the Sequencer and responded that it was nearly always initiated by the Sequencer. The controllers described the coordination as being nearly always helpful in resolving problems, with an average rating of 4.9 on the post-run survey, where 1 was "Not very helpful, 3 was "Somewhat helpful," and 5 was "Very helpful."

American Institute of Aeronautics and Astronautics 
Once the Sequencer informed / coordinated with the Planner about the location of the B757 gap and the Planner updated the schedule to reflect the change, the schedule and the associated TSS slot markers "jump" to the new locations associated with the new STAs. A proper coordination between the Planner, Sequencer, and controllers is required so that controllers could anticipate the change and manage to the new schedule as soon as it happens. In the study, controllers reported that they were "always" informed about the schedule changes in advance. The post-run question was "If there were any schedule changes that affected you, were you informed about these changes in advance?" A 1-5 rating scale was provided which ranged from $1=$ Never, to $5=$ Always. All controllers answered this question with $5 \mathrm{~s}$, or "Always."

Due to the extra task load associated with conforming to the TSS/DSAS schedule in the TRACON, there was a question as to whether TRACON Sequencer will be more critical for TSS and/or DSAS operations than Baseline, especially for the B757 departure operation. When participants were asked in the post-sim survey question, "About how much do you think a TRACON Sequencer was needed in the following conditions?", surprisingly the controller and Sequencer participants thought the Sequencer was needed most in the Baseline conditions. Figure 15 shows the answers that ranged from 1 to 5 with 1 being "Not at all," 3 "Somewhat," and 5 "Very much." (Not significant). The Planner also thought the Sequencer was needed most in Baseline (ratings were all 5s) but rated this item as 4s in the other conditions, indicating that he thought the Sequencer was useful at all times. Final reported the most coordination with the Sequencer.Error! Reference source not found. The controllers were asked in the post-run survey, "In this run, about how many times did the TRACON Sequencer coordinate with you to achieve appropriate spacing or scheduled times?" The ratings were on a 1-5 scale were as follows:Error! Reference source not found. (Controller Ms: Final 3.3, Empyr 2.2, Haarp 2.2. $M S$ 2.7, $F(2,6)=24, p=.01$ ).

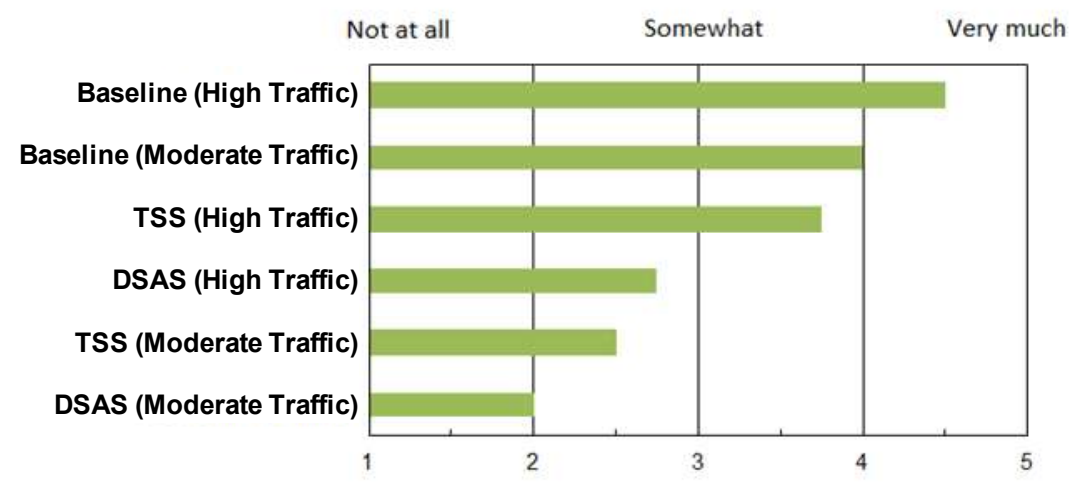

Figure 15. Participant ratings on the importance of Sequencer across the three operational conditions

As shown in Figure 16, the controllers reported they had the most coordination with the Sequencer in Baseline in the High traffic condition. (Tool $\mathrm{x}$ traffic interaction $M S .67, F(2,4)=8.0, p=.04$.) Final reported most of the coordination with the Sequencer especially in the Baseline condition. Controller x Operations $M S .81, F(4,6)=7.3$, $p=.02$. (The operational conditions were not significantly different from each other.)

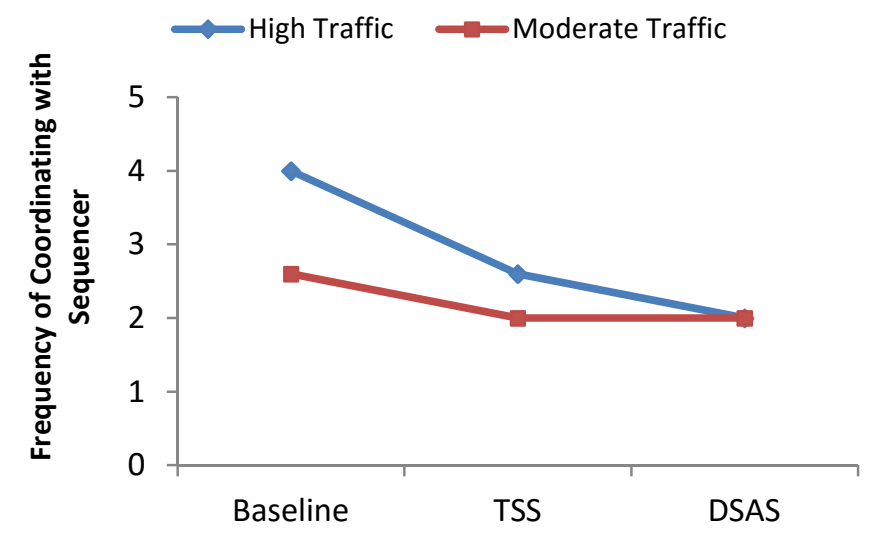

Figure 16. Frequency of coordination with Sequencer across conditions

American Institute of Aeronautics and Astronautics 
In terms of difficulty in creating the gaps for the B757s, when the TRACON controllers were asked in the postrun survey, "In this run, how difficult was it for you to create the required spacing for the 757 departures?", all three TRACON controllers reported least difficulty with the DSAS condition, but their responses diverged when comparing Baseline and TSS. The Final controller reported having the more difficulty spacing $757 \mathrm{~s}$ in Baseline than TSS, Empyr sector controller had less, and Haarp reported the same level of difficulty. The answers ranged from 1 to 5 with a being "Not very difficult, 3 "Somewhat difficult," and 5 "Very difficult." (Controller X tools MS .72, $F(4,6)=13, p<.01$; controller $M s$ Final 1.8, South Feeder 1.2, North Feeder 1.7, $F(2,6)=13, p=.01$.)

\section{F. Controller Workload and Task load Distribution}

For TSS and/or DSAS operations to be feasible, it cannot create workload that is infeasible to the controllers. TSS and DSAS were expected to potentially reduce controller workload, especially for the Final controller by using the terminal scheduler to manage the arrival spacing and merging seamlessly and across multiple sectors. Conversely, the TSS/DSAS operations also had a potential for increasing controller workload, especially in the B757 departure scenarios, when the schedule needed to be modified and the adjustment required significant coordination and re-planning. The following section examines the task load associated with clearances given to the aircraft across the conditions, as well as the participants' subjective ratings of workload.

\section{Clearances per Aircraft}

Figure 17 shows the average number of clearances issued per aircraft across the conditions. Two main effects influences the number of clearances issued per aircraft - there here were more clearances issued in Baseline $(M=$ 8.0, $S D=5.4)$, than in TSS $(M=5.3, S D=4.1)$ or DSAS $(M=4.3, S D=3.0), F(2,258)=18.3, p=.000$. This result suggests that Baseline runs required more control to deliver aircraft to the runway, than when controllers had slot markers and timelines. There were also more clearances issued in High traffic $(M=6.7, S D=4.6)$ than in Moderate traffic $(M=5.0, S D=4.4), F(1,258)=11.0, p=.001$. This suggests that 6 additional aircraft $(15 \%$ more $)$ in the High traffic condition reduced the capacity enough to significantly increase the workload for controller in managing sequencing and spacing aircraft.

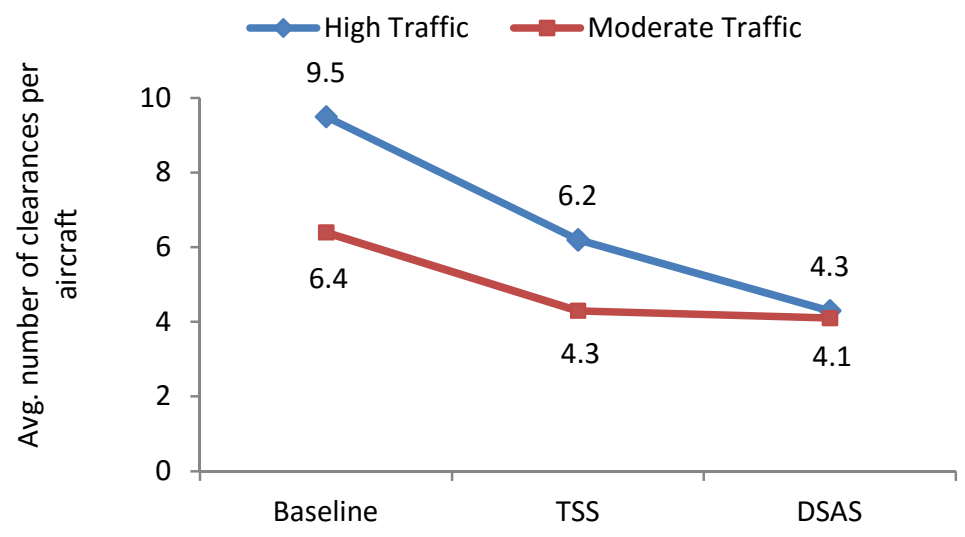

\section{Figure 17. Average number of clearances per aircraft as a function of traffic and operational conditions}

There was a marginal interaction effect of Traffic and Operational Environment, $F(2,258)=2.6, p=.08$. It seemed that the difference between High and Moderate traffic decreased progressively from Baseline $(M=9.5$ vs $M$ $=6.4)$, to TSS $(M=6.2$ vs $M=4.3)$, and to DSAS $(M=4.3$ vs $M=4.1)$ where there was no difference. In Moderate traffic condition, Baseline was higher than TSS and DSAS, which were also identical $(M=4.3 \& M=4.1$, respectively). Four clearances per aircraft in the most efficient conditions seem to be minimal given the comparatively high demand in Moderate traffic (35 per hour). Higher traffic level may have left less slack in the schedule and may have required more adjustment per aircraft to deliver with minimum spacing at the runway. This reduced capacity may also have been more impacted when spacing needed to be created for heavies in Baseline and TSS runs.

American Institute of Aeronautics and Astronautics 


\section{Workload Ratings by Traffic Level and Operational Condition}

Participants were asked to report their workload ratings during and after each simulation run. Follow up questions related to workload were also asked in the post-sim survey. The overall results mirror the task load as measured in the number of clearances, albeit with some minor differences.

Post-run ratings of mental activity at the busiest time decreased as the tools became more advanced in the three operations (see Figure 18). Controller ratings were in response to the question, 'In the last run, how much mental activity was required during the busiest time?" $(M=3.3,2.0$ and 1.2 for Baseline, TSS and DSAS, respectively, $F(2,4)=17.2, p=.01)$. Reported mental activity also increased in the higher traffic condition $(M=1.7$ and 2.7 for Moderate and High traffic, respectively, $F(1,2)=27, p=.04)$.

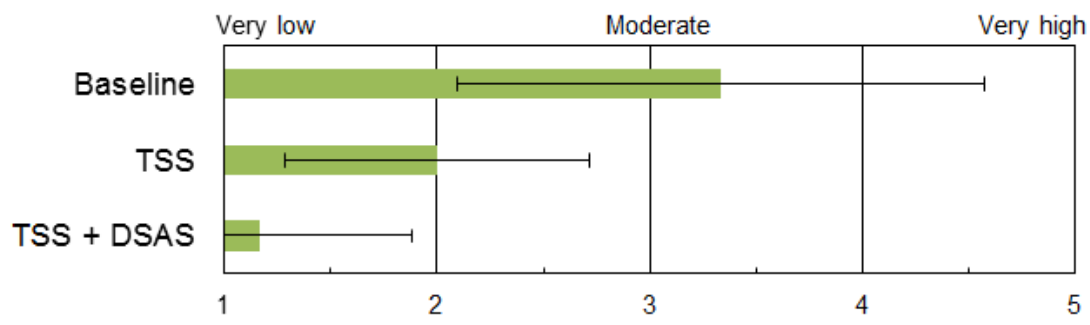

Figure 18. Post-run ratings of mental activity at the busiest time decreased with more advanced tools. Error bars are $95 \%$ CIs

Similar to the clearance data in Figure 17, the subjective ratings on mental activity was progressively less with higher tool capability and High traffic resulted in higher mental activity than Moderate traffic. Also, there were interaction effect between Traffic and Operations (Traffic x Operations Interaction, $F(2,4)=1.2, p=.02$.) but the effect was much stronger, mainly due to relatively high mental activity for TSS in the High traffic condition.

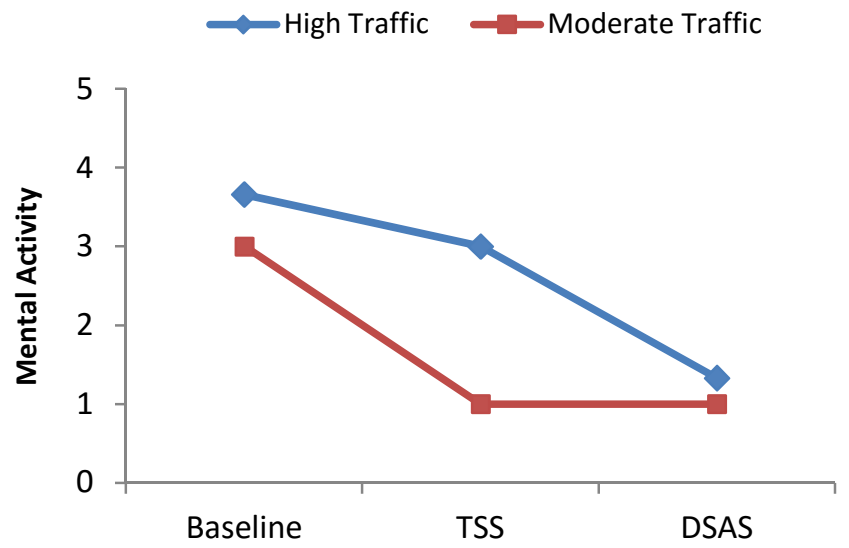

Figure 22. Post-run ratings of mental activity across conditions

In the post-sim survey, when the controllers and TRACON sequencer were asked to "Please rate your typical workload in the following conditions," they indicated that they experienced heavy workload in the High traffic Baseline condition and moderate workload in the Moderate traffic Baseline condition (see Figure 23). In the other conditions, the workload was described as much lighter - in the Moderate traffic DSAS condition even approaching "Not enough to do." In these post-sim ratings, TSS was also seen as reducing the workload somewhat in High traffic conditions, but not as much as DSAS. A repeated measures analysis of variance on these ratings indicates that both condition and traffic levels were significantly different. $\quad(M=3.8,2.1,1.6$ for Baseline, TSS and DSAS, $F(2,6)=7.5, p=.02$. $M=2.0$ and 3.0 for Moderate and High traffic, $F(1,3)=25.0, p=.04)$.

American Institute of Aeronautics and Astronautics 


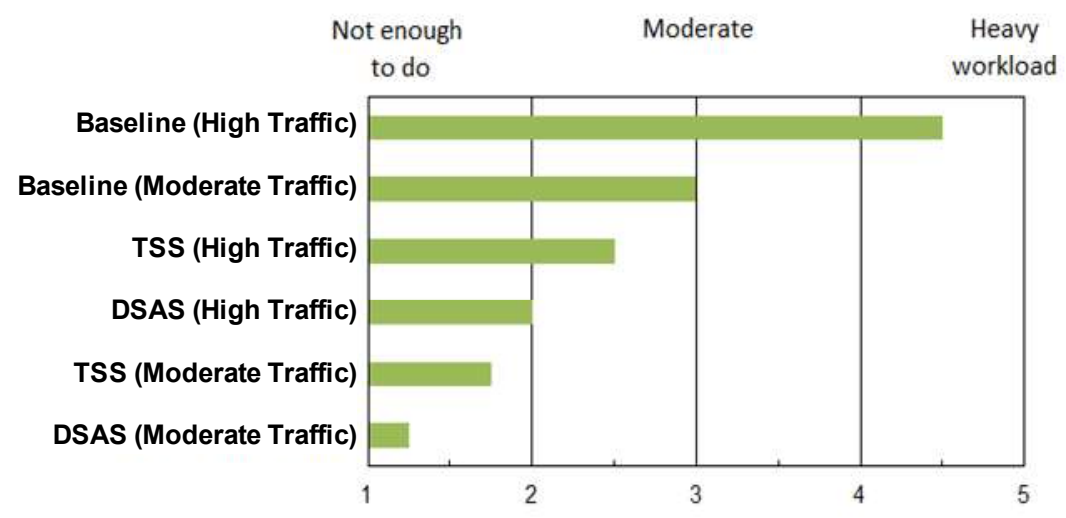

Figure 23. Post-sim workload ratings across conditions

\section{Task Load / Workload Distribution}

The TSS/DSAS conditions also influenced the distribution of the task load across sectors. Table 9 shows the distribution of clearances across sectors for each tool condition in High traffic runs. As can be seen, there was a shift in task load between the Final and the feeder sectors, Empyr and Haarp. In the Baseline run, Final issued 59.6\% of the clearances (236/396), compared to 25.5\% (101/396) by Empyr and 14.9\% (59/396) by Haarp. In contrast, in the TSS run, Final issued $14.6 \%$ of the clearances (36/247), compared to $55.4 \%$ (137/247) by Empyr and $30.0 \%$ (74/247) by Haarp, showing the shift in the task load distribution, with fewer clearances by Final but more by the feeder sectors compared to Baseline. Similarly in the DSAS run, Final issued 3.6\% of the clearances (6/166), compared to $56.0 \%(93 / 166)$ by Empyr and $40.4 \%(67 / 166)$ by Haarp. $\chi^{2}(4)=226.8, p<.0001$.

Table 9. Clearances per sector in High traffic condition

\begin{tabular}{|l|c|c|c|c|}
\hline Clearances & \multicolumn{1}{|c}{ Empyr } & \multicolumn{1}{c|}{ Haarp } & Final & Total \\
\hline Baseline High & $\begin{array}{c}101 \\
(25.5 \%)\end{array}$ & $\begin{array}{c}59 \\
(14.9 \%)\end{array}$ & $\begin{array}{c}236 \\
(59.6 \%)\end{array}$ & $\begin{array}{c}396 \\
(100 \%)\end{array}$ \\
\hline TSS High & $\begin{array}{c}137 \\
(55.5 \%)\end{array}$ & $\begin{array}{c}74 \\
(30.0 \%)\end{array}$ & $\begin{array}{c}36 \\
(14.6 \%)\end{array}$ & $\begin{array}{c}247 \\
(100 \%)\end{array}$ \\
\hline DSAS High & $\begin{array}{c}93 \\
(56.0 \%)\end{array}$ & $\begin{array}{c}67 \\
(40.4 \%)\end{array}$ & $\begin{array}{c}6 \\
(3.6 \%)\end{array}$ & $\begin{array}{c}166 \\
(100 \%)\end{array}$ \\
\hline Total & 331 & 200 & 278 & 809 \\
\hline
\end{tabular}

Table 10 shows the distribution of clearances across sectors for each tool condition in Moderate traffic runs. Similar to the High traffic runs, there was a shift of task load between Final and the feeder sectors, Empyr and Haarp. In Baseline, Final issued 57.3\% of the clearances (145/253), compared to 29.6\% (75/253) by Empyr and to $13.0 \%$ (33/253) by Haarp. In TSS, Final issued 6.1\% (10/164) of the clearances, compared to $68.3 \%(112 / 164)$ by Empyr and $25.6 \%(42 / 164)$ by Haarp. In DSAS, Final issued $6.9 \%$ of the clearances (9/131), Empyr issued $64.1 \%$ $(84 / 131)$, and Haarp issued $29.0 \%(38 / 131) \cdot \chi^{2}(4)=168.8, p<.0001$.

Table 10. Clearances per sector in Moderate traffic condition

\begin{tabular}{|l|c|c|c|c|}
\hline Clearances & \multicolumn{1}{|c|}{ Empyr } & \multicolumn{1}{c|}{ Haarp } & Final & Total \\
\hline Baseline Mod & $\begin{array}{c}75 \\
(29.6 \%)\end{array}$ & $\begin{array}{c}33 \\
(13.0 \%)\end{array}$ & $\begin{array}{c}145 \\
(57.3 \%)\end{array}$ & $\begin{array}{c}253 \\
(100 \%)\end{array}$ \\
\hline TSS Mod & $\begin{array}{c}112 \\
(68.3 \%)\end{array}$ & $\begin{array}{c}42 \\
(25.6 \%)\end{array}$ & $\begin{array}{c}10 \\
(6.1 \%)\end{array}$ & $\begin{array}{c}164 \\
(100 \%)\end{array}$ \\
\hline DSAS Mod & $84(64.1 \%)$ & $\begin{array}{c}38 \\
(29.0 \%)\end{array}$ & $\begin{array}{c}9 \\
(6.9 \%)\end{array}$ & $\begin{array}{c}131 \\
(100 \%)\end{array}$ \\
\hline Total & 271 & 113 & 164 & 548 \\
\hline
\end{tabular}

22

American Institute of Aeronautics and Astronautics 
Figure 24 graphically represents the clearances. Each dot in the graph indicates an altitude, speed, or heading clearance by the controllers. The graphs show that the Final controller, who issued many clearances in the Baseline condition, issued very few clearances in the TSS and DSAS conditions. In contrast, the Empyr sector issued many more speed clearances in the TSS and DSAS conditions compared to Baseline. The results suggest that the TSS/DSAS tools redistributed the task load across the sectors, making the problem much easier to manage for the controllers as a whole. The findings were supported from the subjective ratings of the workload, which mirrored the task load data shown here.

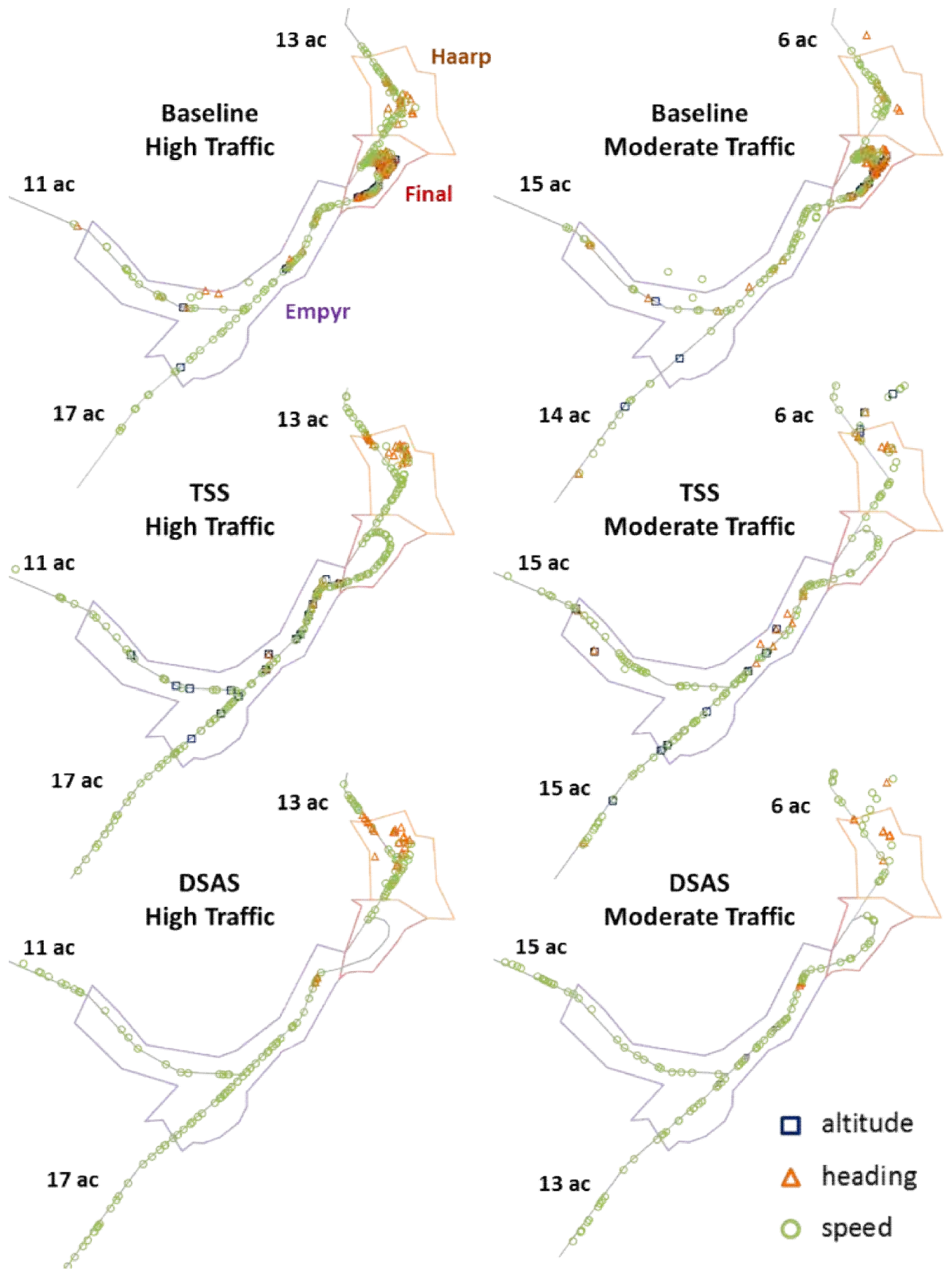

Figure 24. Task load distribution across Empyr, Haarp, and Final sectors 
Similar interactions between the Final and the feed sectors were present in the subjective workload ratings. For workload acceptability across all conditions, the TRACON controllers rated the workload in the post-run surveys as "Very acceptable" (fives) in all runs, with the exception of Baseline conditions in which the ratings were between 3 and 4. Breaking the ratings down by controller, Final controller had an average rating of two in the Baseline condition, which is what reduced the average ratings in that condition. For Final, compared to the other controllers, the workload was not acceptable in the Baseline conditions. (Final $M$ 2.0, Empyr $M$ 5.0, Haaro $M=4.8, M S$ 1.7, $F(2,6)=31.0 p=.01$. Controller x Operations $M S 1.7, F(4,6)=31, p=.01$. Baseline $M 3.8$, TSS $M$ 5.0, DSAS $M$ $5.0, M S .91, F(2,3)=49.0, p=.01)$.

Other measures of workload were the number of times controllers reported interacting with the pilots to change aircraft speed or altitude, clear the aircraft" direct to fix," and vector the aircraft. On the post-run survey, controllers were asked the question, "In this run, about how many times did you use the following methods to control the aircraft in your sector?" The ratings were on a 1-5 scale with 1 = "Never," 2 = "About 1-3 times," 3 = "About 4-7 times," 4 = "about 8-10 times," and 5 = "More than 10 times." When asked how frequently the controllers changed speed of aircraft in their sector in the three tool conditions, Final controller reported using speed much more frequently in the Baseline condition. (Controller $M s=$ Final 3.0, South Feeder 4.8, North Feeder 4.0, MS 5.1, F(2,6) $=11.4, p<.01$; Controller x Operations interaction, $M S 3.0, F(4,6)=6.7, p=.02$ )

Final controller reported changing altitude much more in the Baseline condition. (Controller $M \mathrm{~s}$ Final 2.3, South Feeder 2.8, North Feeder 4.0, $M S 4.4, F(2,6)=8.8, p=.02$; Controller x Operations interaction, $M S 4.9, F(4,6)$ $=9.8, p<.01$.) The Final controller cleared the aircraft "direct to fix" in their sector in the three tool conditions. As can be seen, Final did so much more in the Baseline condition. (Controller Ms Final 2.3, South Feeder 2.8, North Feeder 4.0, $M S$ 4.4, $F(2,6)=8.8, p=.02$; Controller x Operations interaction, $M S 4.9, F(4,6)=9.8, p<.01$.) Finally, the Final controller reported vectoring aircraft more frequently in the Baseline condition. (Controller Ms Final 2.3, South Feeder 1.7, North Feeder 3.8, MS 7.4, $F(2,6)=44.3, p<.001$; Controller x Operations interaction, $M S$ 4.6, $F(4,6)=27.3, p=.001$.

The controller also rated their workload during the simulation using the Workload Assessment Keypad (WAK) were low overall. Unlike the post-run and post-sim ratings did not differ by the Operations condition, but they did differ by Traffic condition ( $M=1.7$ and 2.3 for Moderate and High traffic, respectively, $F(1,2)=97.4, p=.01)$. The average WAK peaks were also different between low and high traffic $(M=2.7$ and 3.2 for Moderate and High traffic, respectively, $F(1,2)=25.0, p=.04)$. However, there was an interaction effect between Controller and Operational Environment (Controller X tool interaction $M S .14, F(4,6), p=.02$ ). Mirroring the clearance data, the Final controller reported higher workload for Baseline compared to TSS while Empyr reported higher workload for TSS than Baseline. The results suggest that the workload was re-distributed from the Final to the Empyr sector in the TSS and DSAS tools conditions.

\section{Arrival Schedule Conformance Management by Feeder Sectors across Conditions}

In the previous section, the distribution of clearances and subjective feedback suggest a re-distribution of workload from Final in the Baseline condition to the Empyr sector in both TSS and DSAS conditions. Figure 24 illustrates visually that in TSS and DSAS conditions, the schedule conformance seemed to be completed mostly by the feeder sectors, requiring very few clearances from the Final sector controller.

In order to verify this observation, the schedule conformance data were analyzed at the TRACON entry point, as an input traffic condition before any scheduling conformance is done and at the entry point of the Final sector, as a way of calculating the schedule conformance performed by the feeder sectors. TRAC (TCSim Route Analyzer/Constructor) was used to acquire the scheduling conformance data ${ }^{12}$. TRAC allows the user to draw "control gates" which could be used to generate the ETAs and STAs at the runway timelines when aircraft crosses these gates, which were drawn at the TRACON and the Final sector entry points. As each aircraft crossed the sector boundary, its schedule conformance was calculated as the difference between the most current ETA for the runway threshold at the crossing point minus the last assigned STA for the threshold. Using this definition, a positive value indicated that the aircraft was late; a negative value indicated that the aircraft was early, and a zero value indicated that the aircraft was on time.

Figure 25 illustrates the schedule conformance across the three Operational conditions for the TRACON and Final sector entry points. For the Baseline condition, the distribution of the conformance for the TRACON entry and the Final sector is similar as expected, since the controllers were not managing towards a schedule. In the TSS and DSAS conditions, the left graphs showing the traffic at the TRACON entry have a broad distribution similar to

American Institute of Aeronautics and Astronautics 
Baseline, but by the time that the aircraft enters the Final sector, the right graphs show that the scheduling performance delivery precision were quite good. In these conditions, over $82 \%$ for TSS and $92 \%$ for DSAS condition of aircraft were conforming to the schedule within \pm 10 seconds (section -10 and 0 of $x$-axis) when the aircraft entered the Final sector, suggesting that the Final controller had very little scheduling work to do.

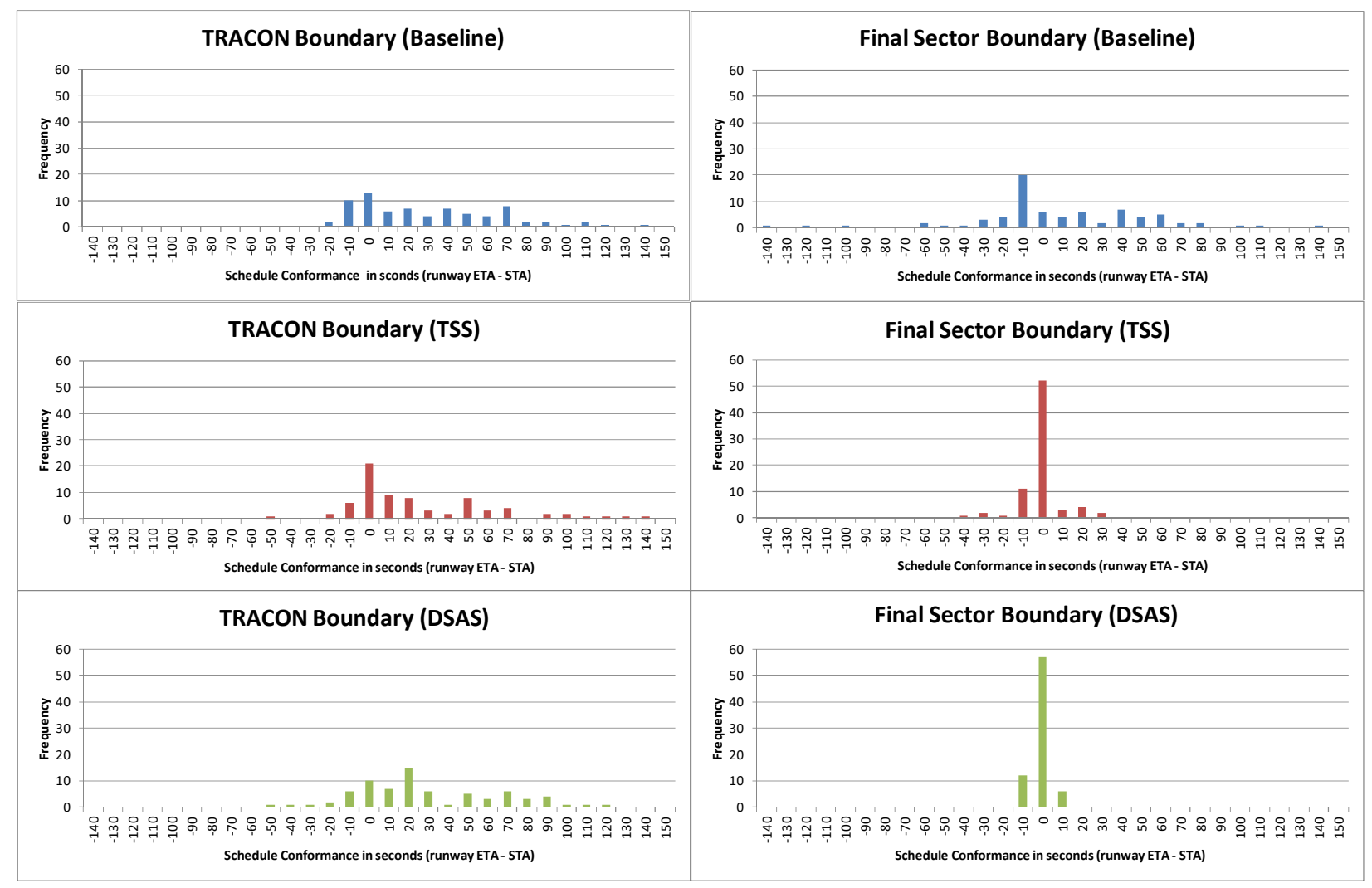

Figure 25. Schedule conformance at the TRACON and Final sector entry points. Positive value = late; negative value $=$ early. The number on the $x$-axis $=$ the lower bound of the bin: $e . g .-10=[-10,0]$

Examining the data more closely, the majority of the conformance data lie between \pm 20 seconds and most of the outliers (bigger than \pm 30 seconds) were caused by adjustments for the 757 departures. Levene's test for equality of variances were applied to the conformance data. For the Moderate traffic level, there was no significant difference in variance for the schedule conformance between Baseline, TSS, and DSAS conditions when the aircraft entered the TRACON entry point as expected $(F(2,101)=1.03, p=.36)$ but after the controllers managed the traffic according to the Baseline, TSS and/or DSAS operations, the schedule conformance at the runway threshold was significantly different across the conditions, $F(2,101)=26.29, p<.01$. Similarly for the High traffic levels, there were no significance in the schedule conformance variance at the TRACON entry across conditions (Levene's test for equality of variances, $F(2,117)=.44, p=.64)$ but found a significant difference between the conditions at the runway threshold, $F(2,117)=28.54, p<.01$.

\section{G. Safety}

During the six data collection runs, the three TRACON controllers were asked to deliver the arrivals in-trail to runway 22. In the simulation, the Final controller was asked to deliver the arrivals with minimum $2.5 \mathrm{~nm}$ in-trail spacing close to the runway before handing them off to the Tower controller. For the feeder sectors, i.e. Haarp and Empyr, the controllers were asked to maintain the TRACON separation rules, including maintenance of $3 \mathrm{~nm}$ minimum separation for non-divergent aircraft.

Based on those criteria, there were three separation violations recorded during the runs. All three violations occurred in the Baseline condition, two in the Final sector and one in the Haarp sector. Two violations in the Final sector occurred, one each in High and Moderate traffic conditions. The violation in the Haarp sector occurred in the High traffic condition. The closest point of approach between the aircraft pairs were $2.42 \mathrm{~nm}$ and $2.33 \mathrm{~nm}$ for the 
two violations in the Final sector in High and Moderate traffic conditions, respectively. The closest point of approach was $2.24 \mathrm{~nm}$ for the Haarp sector. No violations occurred during the TSS or DSAS conditions.

Figure 26 illustrates the instance when the separation violations were initially detected in Final (left figure) and Haarp (right figure) sectors. All three violations in Baseline occurred when aircraft were vectored off of their routes to create the necessary in-trail spacing but were merged back into the flow too tightly to the leading aircraft that violated the minimum spacing.
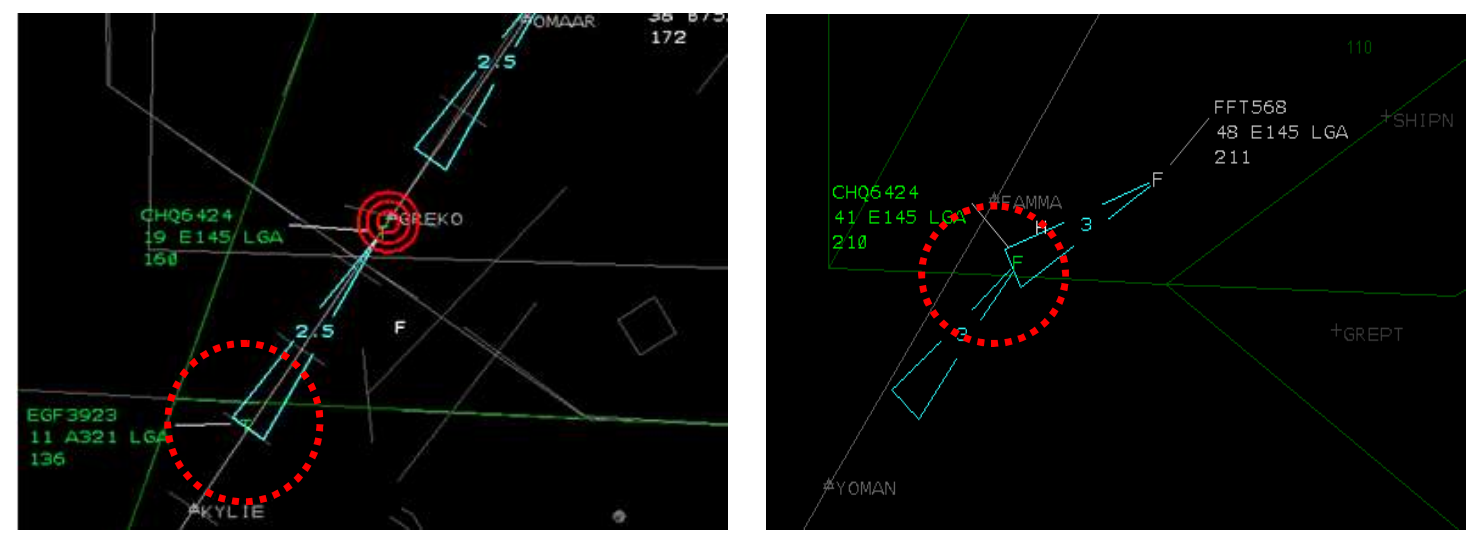

Figure 26. Box and whisker plots for the Moderate (left) and High Traffic Levels (right)

When the participants were asked about the safety of the different conditions, the seven participants (the three TRACON Controllers, Sequencer, Planner, Cab Coordinator, and Tower) indicated that safety was acceptable in all six runs, but most acceptable in the DSAS condition. After each run, participants were asked, "In this run, how acceptable in terms of safety were operations [in your sector/in the Tower/in the TRACON/that you (the Planner) saw]?" All participant ratings were either fours or fives on a scale of 1 to 5 (see Figure 27). However, in the DSAS condition, all of the ratings were fives, baseline $M=4.7$, TSS $M=4.9$; DSAS $M=5.0, F(2,12)=4.5, p=.035$. There was no difference in safety ratings by traffic condition.

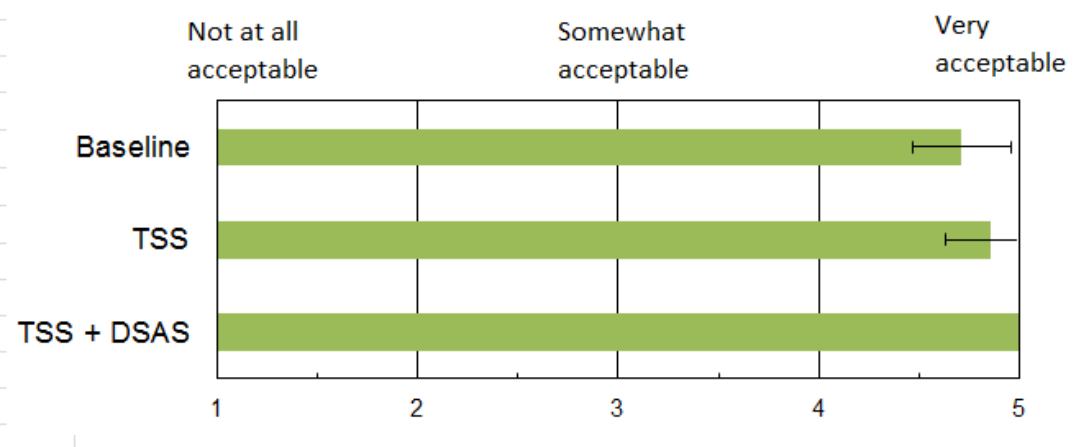

Figure 27. Post-run ratings of safety acceptability were slightly higher in the TSS + DSAS condition. Error bars are $95 \%$ CIs.

Participants were asked to give additional feedback with the question "If operations were "somewhat acceptable' or less for any reason, in what regard and why?" The Final controller, who had indicated that operations were only "somewhat acceptable" (threes) in regard to safety responded, "Baseline conditions were much more difficult than TSS and DSAS. The tools took away the need for me to plan ahead to make the appropriate arrival and departure holes."

\section{H. Efficiency}

American Institute of Aeronautics and Astronautics 


\section{Lateral Path Deviations}

Without the TSS/DSAS scheduler in the Baseline condition, the upstream feeder sectors (i.e. Empyr and Haarp) did not coordinate their respective feed to a common merge point in the Final sector, which in turn resulted in Final controller to vector the arrivals to sequence them before landing. Figure 28 shows the track plots of the traffic in each of the 6 runs. As expected the two Baseline runs show more lateral deviations from the route in the Final sector than in other conditions (TSS and DSAS). The high traffic runs show an increase of deviations in Haarp sector compared to the low traffic runs, which was also expected since Haarp had less traffic and more space would be vectoring more traffic than Empyr to help the last merge in Final airspace. Overall, Empyr controller seemed to have kept traffic on its route the most, compare to Final and Haarp controllers.

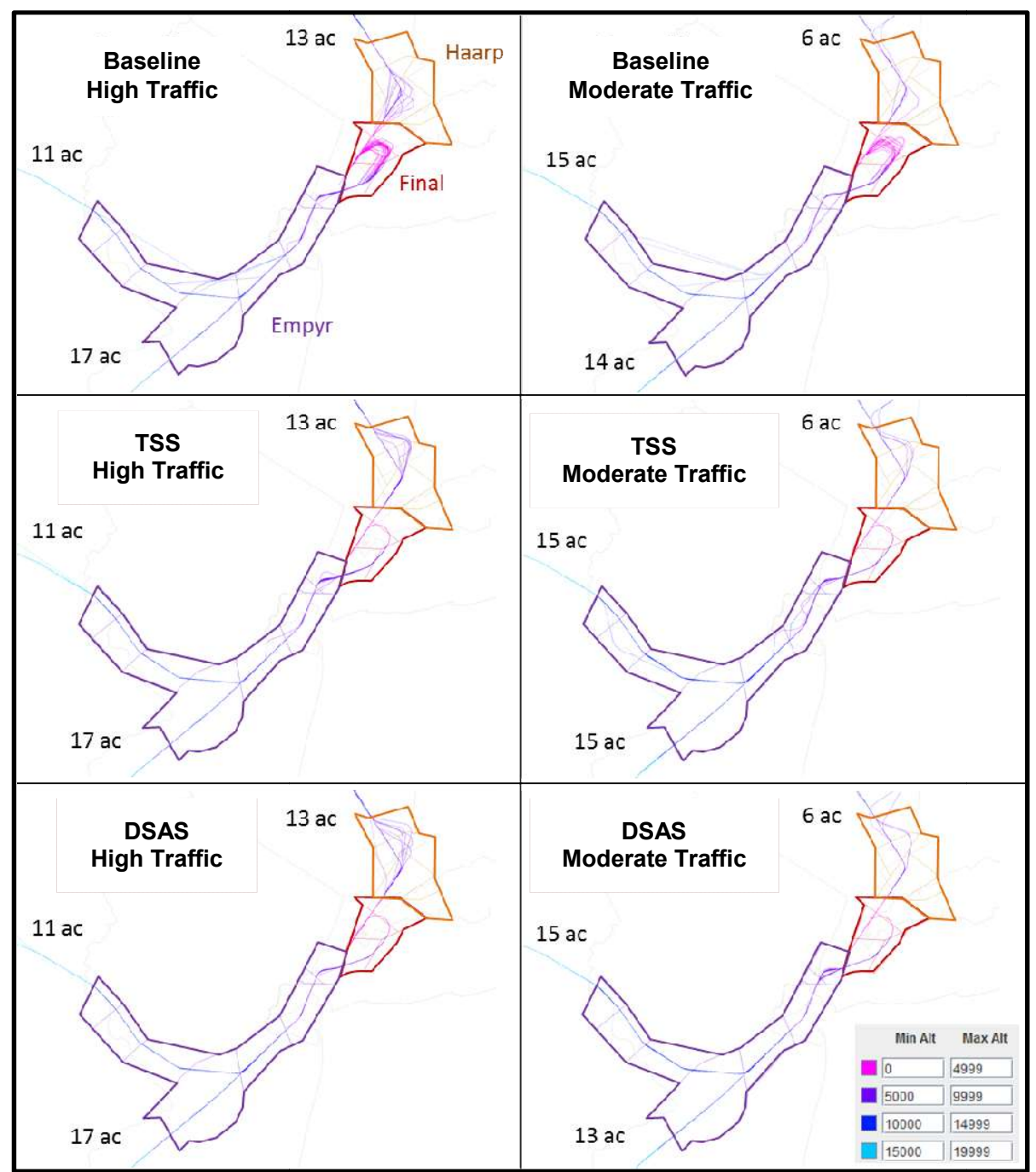

Figure 28. Tracks of traffic in each runs with the number of aircraft (ac) from each entry point

\section{Flight Distance and Time}

Figure 29 displays the total distance flown in nautical miles $(\mathrm{nm})$ in each run as a function of conditions and sectors in the TRACON airspace. A difference in flown distance was expected between High and Moderate traffic conditions, as well as between the Baseline and TSS/DSAS due to potential increase in vectoring. However, results showed no noticeable differences in the total distance flown across all sectors. Also, contrary to expectations, the total flight path totaled to a lesser amount $(3147 \mathrm{~nm}$ and $3120 \mathrm{~nm}$ ) in Baseline compared to other runs (3249nm in TSS High, $3240 \mathrm{~nm}$ in DSAS High, and $3235 \mathrm{~nm}$ in TSS Moderate). The breakdown by sectors displays similar

American Institute of Aeronautics and Astronautics 
consistency across the runs, with the exception of Haarp. There was less flight distance covered in Haarp in the Moderate traffic runs than in the High traffic runs. Similarly, Empyr had a slight increase of flight distance in the Moderate traffic runs. The main reasons for these two differences is due to the traffic scenarios, where Haarp has 6 aircraft in the Low traffic runs (7 less than in the high traffic runs), and Empyr has 1 or 2 more aircraft in the Low traffic (28-29) than in the High traffic runs (28).

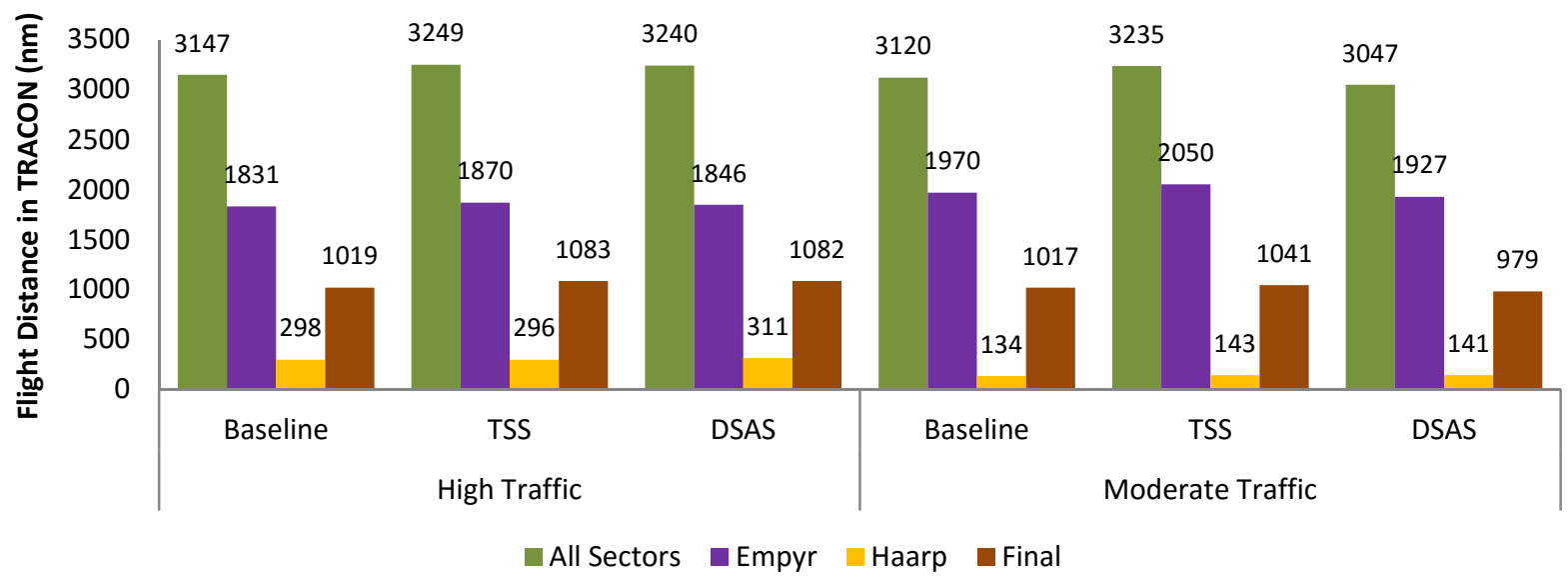

Figure 29. Sum of flight distance in TRACON airspace across all runs per conditions and sectors

Similarly, Figure 30 displays the total time in seconds flight spent in the TRACON airspace as a function of conditions and sectors, which also shows no discernible difference in flight time across the three experimental conditions. The total time in the High traffic runs shows an $8 \%$ increase of flight time $(819 \mathrm{~min})$ compared to the Moderate traffic runs $(755 \mathrm{~min})$. There are twice as less flight time in Haarp sector in the Moderate traffic runs than in the High traffic runs, in conformance with the number of aircraft in Low (6) versus High (13) traffic level. It is also worth mentioning that aircraft spent nearly the same amount of time in Final sector as in Empyr sector, despite that routes in Empyr are significantly longer. This is due to aircraft slowing down as they approach to the runway.

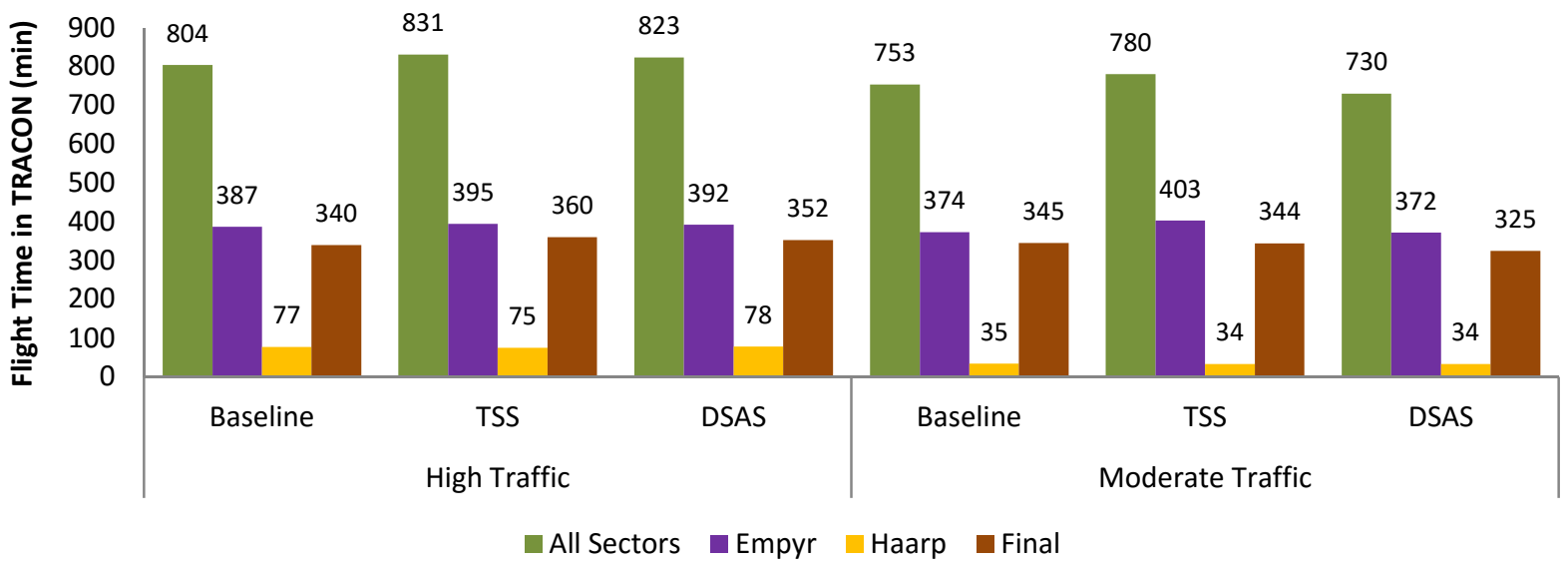

Figure 30. Sum of flight time in TRACON airspace across all runs per conditions and sectors

\section{Discussion}

LaGuardia (LGA) departure delay is a serious bottleneck caused by an inter-dependency between LGA's arrival and departure runways and high persistent arrival demand that result in missed departure slots which aggregate over the day and create significant departure delays and taxiway congestion. In this paper, TSS and DSAS concepts were evaluated for LGA operations to see if they can increase departure throughput and reduce delays.

American Institute of Aeronautics and Astronautics 
The throughput results suggest that with a moderate arrival traffic demand, it seemed fairly easy to depart a greater number of aircraft than arrivals for both Baseline and TSS conditions without adjusting the arrival schedule. With DSAS operations and precise arrival metering to ensure departure gaps, they were able to get 4 or 5 more departures out over the Baseline or TSS conditions, which could be valuable in situations in which a significant departure queue exists at the airport. In the moderate traffic scenarios, TSS schedule alone did not seem to show benefits, mainly because there seemed to be enough slack in the arrival schedule such that the imprecise inter-arrival spacing in the Baseline condition did not adversely create missed slots.

The high traffic condition was where the operational benefits of both TSS and DSAS can be seen. In the Baseline condition, the controllers were unable to maintain one-for-one departure/arrival operation (41 arrivals vs. 38 departures) because inconsistent arrival spacing led to missed departure slots. By creating and managing to a consistent arrival schedule in TSS, the controllers were able to depart six more aircraft than Baseline. Furthermore, adjusting the arrival schedule in the DSAS condition allowed for nine more departures to be release than the Baseline condition. The detailed look at the number of missed departure slots and the recovered extra departures seem to confirm that the benefits in the high traffic condition for TSS and DSAS were mainly due to preventing the missed departure slots, whereas the benefits in the moderate traffic for DSAS was mainly due to optimized arrival scheduling. The increased departure throughput directly impacts the reduction in departure delays, as the results showed approximately 10 minutes of delay reduction for each departure for high traffic DSAS condition. Since the high arrival demand persist throughout the day at LGA, the time savings has a potential to be very substantial.

The inter-arrival spacing data showed that DSAS operations indeed were able to cluster the spacing around the target spacing but the impact was very subtle in a way that statistical test did not show the difference across the conditions. The overall difference in the extra departure spacing that were created were just few extra but those small increments played a significant role in reducing the overall delays.

The tactical adjustments of the terminal scheduler in TSS and DSAS conditions performed smoothly with acceptable and feasible procedures between the tower cab coordinator, Sequencer, Planner, and the controllers. The coordination procedures were well accepted and the workload for these conditions were less than Baseline.

The controller workload for TSS and DSAS were lower than the Baseline condition, with DSAS resulting in the lower workload. Examining the clearances across the sectors and conditions, it was clearly evident that TSS and DSAS resulted in much fewer clearances than Baseline. It can be seen that the Final controller issued more heading and altitude clearances in the Baseline than in the TSS and DSAS runs. In fact, in the TSS and DSAS runs, the Final controller issued only speed clearances, and much less often. Comparatively, there seems to be no obvious reduction of speed clearances in the feeder sectors (Empyr and Haarp) across the conditions. This phenomenon can be explained the use of the TSS tools by controllers in both TSS and DSAS conditions. They provide a mean for controllers to keep aircraft in its target position so aircraft could have the required spacing (conformance to STA). In the Baseline condition, controllers did not know whether aircraft were too early or late in regards to their STA. Conversely, in the tool conditions, controllers acted earlier. It seemed that the feeder sectors had issued clearances further outside their sector in the TSS and DSAS condition, than in the Baseline condition. By the same token, it seems that Haarp controller issued heading clearances earlier in its sector in the TSS and DSAS runs than in the Baseline runs. This is particularly the case in the high traffic runs. Speed was mainly used by controllers to space aircraft accordingly, but there seem to be fewer altitude clearances issued in DSAS compared to TSS. This could be due to the fact that there was more frequent rescheduling taking place in TSS than in DSAS runs. As a result, the Empyr controller used more frequently altitude along with speed as a mean to slow or speed up aircraft by keeping aircraft above or below $10,000 \mathrm{ft}$ (speed is limited to $250 \mathrm{knots}$ below 10,000ft).

Finally, the flight time and distance was expected to be improved under TSS and DSAS conditions since they resulted in less vectoring but the results showed no differences across the conditions. There were also no safety issues for the TSS and DSAS conditions, as three separation errors in the study occurred in the Baseline condition.

\section{Conclusion}

Overall, TSS and DSAS operations demonstrated a concept that can improve chronic departure delay problem at LGA. Although TSS alone showed significant benefit to the departure throughput compared to Baseline, especially with very high arrival demand, the DSAS concept provided even greater benefit for both moderate and high arrival demand. TSS and DSAS operations also provided a significant benefit of distributing the arrival management task evenly across multiple sectors and implicitly coordinate arrivals from different feeder sectors to a downstream merge point in a seamless manner.

29

American Institute of Aeronautics and Astronautics 
In summary, both TSS and DSAS operations provide one step towards improving operations at the NY metroplex. The concept was demonstrated to various stakeholders who have shown significant interest in the proposed solution. Although the results suggest benefits that are specific to LGA operations, the concept and the associated benefit mechanisms should be applicable to other airports with similar arrival-departure dependencies. The TSS technology is currently in the FAA's NextGen implementation plan and therefore could be utilized once it is adapted to the LGA arrival routes, and the DSAS tools could be built by adapting the TSS technology. The next step for the concept evaluation would be to test both TSS and DSAS in a more realistic environment with different winds, runway configurations, traffic scenarios, and with off-nominal situations.

\section{References}

${ }^{1}$ Lee, P. U., Smith, N. M., Homola, J., Brasil, C., Buckley, N., Cabrall, C., Chevalley, E., Parke, B. and Yoo, H., "Reducing Departure Delays at LaGuardia Airport with Departure-Sensitive Arrival Spacing (DSAS) Operations." Proceedings of the Eleventh USA/Europe Air Traffic Management Research and Development Seminar, Lisbon, Portugal, 2015.

${ }^{2}$ Diffenderfer, P. A., Zheng, T. and Gaea, P., “Automated Integration of Arrival/Departure Schedules,” Proceedings of the Tenth USA/Europe Air Traffic Management Seminar, Chicago, Illinois, 2013.

${ }^{3}$ D. Bohme, "Tactical departure management with the Eurocontrol/DLR DMAN," Proceedings of the Sixth USA/Europe Air Traffic Management Seminar,, Baltimore, MD, USA, 2005.

${ }^{4}$ National Air Traffic Services, 2014, Building a shared picture for tower and Approach. Retrieved April 26, 2015 from http://nats.aero/blog/2014/10/building-shared-picture-tower-approach/

${ }^{5}$ Swenson, H., Thipphavong, J., Sadovsky, A., Chen, L., Sullivan, C. and Martin, L., "Design and Evaluation of the Terminal Area Precision Scheduling and Spacing System," Proceedings of the 9th USA/Europe Air Traffic Management Research and Development Seminar, Berlin, Germany, 2011.

${ }^{6}$ Thipphavong, J., Jung, J., Swenson, H., Martin, L., Lin, M., and Nguyen, J., "Evaluation of the Terminal Sequencing and Spacing System for Performance-Based Navigation Arrivals," Proceedings of the 32nd Digital Avionics Systems Conference, Syracuse, NY, October 2013.

${ }^{7}$ Yoo, H., Lee, P., and Palmer, E.,"Improving Departure Throughput by Dynamically Adjusting Inter-arrival Spacing," Proceedings of the 33rd Digital Avionics Systems Conference, Colorado Springs, CO, October 2014.

${ }^{8}$ Prevot, T., Lee, P., Callantine, T., Mercer, J., Homola, J., Smith, N. and Palmer, E., "Human-in-the-loop evaluation of NextGen concepts in the Airspace Operations Laboratory," Proceedings of the AIAA Modeling and Simulation Technologies Conference, AIAA 2010-7609, Toronto, 2010.

${ }^{9}$ Cousineau, D. Confidence intervals in within-subject designs: A simpler solution to Loftus and Masson's method. Tutorials in Quantitative Methods for Psychology, 1(1), 42-45, 2005.

${ }^{10}$ Morey, R. D. Confidence intervals from normalized data: A correction to Cousineau (2005). reason, 4(2), 61-64, 2008

${ }^{11}$ Baguley, T. Calculating and graphing within-subject confidence intervals for ANOVA. Behavior research methods, 44(1), 158-175, 2012.

${ }^{12}$ Callantine, T. "TRAC Trial Planning and Scenario Generation to Support Super-Density Operations Studies", Proceedings of the AIAA Modeling and Simulation Technologies Conference, AIAA 2009-5836, Chicago, 2009. 